\title{
FDG-PET Imaging in HIV Infection and Tuberculosis
}

\section{Mike Sathekge, MD, PhD, ${ }^{*}$ Alex Maes, MD, PhD, ${ }^{*,+, \pm}$ and Christophe Van de Wiele, MD, PhD*,§}

\begin{abstract}
The intersection and syndemic interaction between the human immunodeficiency virus (HIV) and tuberculosis (TB) epidemics have global prevalence with devastating morbidity and massive mortality. Using FDG-PET imaging it was shown that in HIV-infected individuals, involvement of the head and neck precedes that of the chest and of the abdomen. The sequence of lymph node involvement observed suggests the existence of a diffusible activation mediator that may be targeted via therapeutic intervention strategies. Furthermore, the degree of FDG uptake proved directly related to viral load and inversely related to CD4 cell count. Available data in acquired immune deficiency syndrome (AIDS)-defining cancers further suggest that FDG-PET/CT imaging may be useful for prognostication of cervical cancer and for identifying appropriate sites for biopsy, staging, and monitoring lymphoproliferative activity owing to HIV-associated Kaposi sarcoma and multicentric Castleman disease. Inversely, in HIV-associated lymphoma, FDG uptake in HIV-involved lymphoid tissue was shown to reduce the specificity of FDG-PET imaging findings, the effect of which in clinical practice warrants further investigation. In the latter setting, knowledge of viremia appears to be essential for FDG-PET image interpretation. Early HIV-associated neurocognitive disorder, formerly known as AIDS dementia complex, proved to be characterized by striatal hypermetabolism and progressive HIV-associated neurocognitive disorder or AIDS dementia complex by a decrease in subcortical and cortical metabolism. In lipodystrophic HIV-infected individuals, lipodystrophy proved associated with increased glucose uptake by adipose tissue, likely resulting from the metabolic stress of adipose tissue in response to highly active antiretroviral therapy. Furthermore, ongoing chronic low-grade infection in arteries of HIV-infected individuals could be depicted by FDG-PET/CT imaging. And there is promising data that FDG-PET/CT in HIV may serve as a new marker for the evaluation of thymic function in HIV-infected patients. In the setting of TB, FDG-PET has proven unable to differentiate malignancy from TB in patients presenting with solitary pulmonary nodules, including those suffering from HIV, and thus cannot be used as a tool to reduce futile biopsy or thoracotomy in these patients. In patients presenting with extrapulmonary TB, FDG-PET imaging was found to be significantly more efficient when compared with CT for the identification of more sites of involvement. Thus supporting that FDG-PET/CT can demonstrate lesion extent, serve as guide for biopsy with aspiration for culture, assist surgery planning and contribute to follow-up. Limited available data suggest that quantitative FDG-PET findings may allow for prediction or rapid assessment, at 4 months following treatment instigation, of response to antituberculostatics in TB-infected HIV patients. These results and more recent findings suggest a role for FDG-PET/CT imaging in the evaluation of therapeutic response in TB patients.
\end{abstract}

I

n 2011, an estimated 34.2 million adults and children were living with human immunodeficiency virus (HIV) or acquired immune deficiency syndrome (AIDS) worldwide ${ }^{1}$;

\footnotetext{
* Department of Nuclear Medicine, University of Pretoria, Pretoria, South Africa. $\nmid$ Department of Nuclear Medicine, AZ Groeninge, Kortrijk, Belgium. ₹Department of Morphology and Medical Imaging, University Hospital Leuven, Leuven, Belgium.

\$Department of Nuclear Medicine, University Hospital Ghent, Ghent, Belgium. Address reprint requests to Mike Sathekge, MD, PHD, Department of Nuclear Medicine, University of Pretoria, Steve Biko Academic Hospital, Private Bag X169, Pretoria 0001, South Africa. E-mail: mike.sathekge@up.ac.za
}

whereas in 2010, 1.1 million had HIV-associated tuberculosis (TB) and 350,000 with HIV-associated TB died. ${ }^{2}$ Tragically, $82 \%$ of the global burden of HIV-associated TB and $71 \%$ of the associated deaths occurred in sub-Saharan Africa, with 10 countries in the southern region of Africa accounting for more than $50 \%$ of cases. ${ }^{1,2}$

HIV primarily infects and kills helper T cells (CD4 ${ }^{+} \mathrm{T}$ cells), macrophages, and dendritic cells. ${ }^{3}$ When $\mathrm{CD}^{+}{ }^{+} \mathrm{T}$-cell numbers decline below a critical level, cell-mediated immunity is lost, and the body becomes progressively more susceptible to opportunistic infections such as TB as well as malignancies. ${ }^{4-6}$ Aside from opportunistic infections and malignancy involving 
the brain, in the later stages of progressive HIV infection, patients may also develop a unique condition labeled HIVassociated neurocognitive disorder (HAND), formerly known as AIDS dementia complex (ADC). ${ }^{6,7}$

HIV and TB, a "cursed duo," are a major public health problem. TB is one of the most virulent opportunistic infections, and it appears early in the course of the HIV infection than other opportunistic infections. As it is one of the first opportunistic infection to appear in HIV-infected people, TB may be one of the earlier signs of HIV infection. ${ }^{8}$

The advent of the highly active antiretroviral therapy (HAART) era has greatly reduced the mortality and incidence of new diagnoses of AIDS-defining illnesses in individuals infected with HIV in many countries. ${ }^{9-13}$ This has led to a paradigm shift in the management of HIV, from that of a highly lethal infection to a chronic condition. Consequently, mortality due to comorbid conditions is becoming more common. In spite of the widespread access to HAART, the incidence of death owing to AIDS-defining illnesses such as TB is still high in some countries, especially when the infection is diagnosed late. ${ }^{14-16}$ Therefore, all the interventions should be monitored with appropriate methods and indicators to constitute an integrated surveillance system of HIV care. Hence, molecular imaging with FDG-PET/CT is needed to provide evidence-based data to optimize quality of care and improve survival.

$\mathrm{PET} / \mathrm{CT}$ is increasingly utilized for nononcologic indications. Increased FDG concentration representing increased cell glycolysis is demonstrated in activated macrophages, lymphocytes, and granulocytes as well as neoplastic cells. ${ }^{17,18}$ FDG-PET detects altered cellular metabolic activity and demonstrates sensitivity for infection in the setting of lymphopenia and neutropenia in which clinical manifestations may be atypical and anatomical abnormality may be diminished and difficult to detect with radiology. ${ }^{19,20}$

This added value FDG-PET/CT would enable this modality to become a reliable tool in this clinical setting. This paper reviews currently available PET studies performed to either improve our understanding of the pathogenesis of HIV-1 infection or assess the value of PET imaging in the clinical decision making of patients with HIV-1 presenting with AIDSrelated opportunistic infections such as TB and malignancies.

\section{Whole-body FDG-PET Findings in Relation to Immunovirological Status}

Available data suggest that HIV binding to resting CD4 $\mathrm{T}$ lymphocytes causes them to home from the blood into lymph nodes (LNs), and during the homing process, they are induced into apoptosis only to secondary signals through the homing receptors. ${ }^{21}$ The clinical correlate of this pathophysiological process is peripheral generalized lymphadenopathy with characteristic LN morphology that precedes tissue involution and ultimately results in loss of superficial LNs in the later stage of disease. ${ }^{22}$ Although FDG-PET is reported to be a sensitive and specific technique in oncologic imaging, it is well known that inflammatory and infectious lesions can cause false-positive results. ${ }^{23-25}$ Various types of inflammatory cells such as macrophages, lymphocytes, and neutrophil granulocytes as well as fibroblasts avidly take up FDG. On activation, resting lymphocytes switch to glycolysis and increase their glucose uptake by approximately 20-fold over 24 hours. ${ }^{26,27}$ LN activation in infectious diseases generates an FDG signal that can be easily discerned from background activity. ${ }^{28}$ Several preclinical and clinical studies have evaluated the ability of FDG-PET as a research tool to identify sites of increased FDG uptake and presumptive HIV replication.

Scharko et a ${ }^{29}$ determined whether FDG-PET/CT could identify activated lymphoid tissues in living rhesus macaques and whether this pattern would reflect the extent of Simian immunodeficiency virus (SIV) infection. PET images from SIV-infected animals were distinguishable from uninfected controls and revealed a pattern consistent with widespread lymphoid tissue activation. Significant FDG accumulation in colon along with mesenteric and ileocecal LNs was found in SIV infection, especially during terminal disease stages. Areas of elevated FDG uptake in the PET images were correlated with productive SIV infection using in situ hybridization as a test for virus replication. FDG-PET images of SIV-infected animals correlated sites of virus replication with high FDG accumulation. Fewer tissues had high FDG uptake in terminal animals than midstage animals, and both were clearly distinguishable from uninfected animal scans. Wallace et $\mathrm{al}^{30}$ extended this study in the same animal model to describe lymphoid tissue activation, using FDG-PET. Within a few days after primary infection by intravenous, intrarectal, or intravaginal routes, FDG-PET imaging revealed a distinct pattern of lymphoid tissue activation centered on axillary, cervical, and mediastinum LNs. Increased tissue FDG uptake preceded fulminant virus replication at these sites, suggesting that a diffusible factor of host or viral origin was responsible for lymphoid tissue changes.

In humans, Scharko et a ${ }^{31}$ performed FDG-PET imaging in 15 patients suffering from HIV-1. They observed distinct lymphoid tissue activation in the head and neck during acute disease, a generalized pattern of peripheral LN activation at midstages, and involvement of abdominal LNs during late disease, suggesting that lymphoid tissues are engaged in a predictable sequence. Iyengar et al ${ }^{32}$ investigated the ability of FDG-PET to measure the magnitude of LN activation in 12 patients recently infected with HIV-1 $(<18$ months since seroconversion) and 11 patients with chronic long-term HIV-1 who had stable viremia by real-time polymerase chain reaction (nonprogressors). All patients were asymptomatic. The authors also assessed PET response in 8 HIV-1 uninfected individuals who had received licensed killed influenza vaccine. A summed PET signal was derived from nodes with viral load by linear regression on log-transformed values. Node activation was more localized after vaccination than after HIV-1 infection. In early and chronic HIV-1 disease, node activation was greater in cervical and axillary than in inguinal and iliac chains and summed PET signal correlated with viremia across a 4-log range. Nonprogressors had small numbers of persistently active nodes, most of 
which were surgically accessible. As shown by our group, the FDG signal derived from FDG-PET imaging in patients with HIV not only correlates with viral load but also inversely with CD4 cell count. ${ }^{33}$ Brust et $\mathrm{al}^{34}$ evaluated FDG biodistribution visually and quantitatively in 5 groups: HIV-negative individuals, HIV-positive patients with early infection, HIV-positive patients with advanced disease, HIV-positive patients with suppressed viral loads, and HIV-positive patients who stopped HAART. Healthy HIV-infected patients with suppressed viral loads and HIVnegative individuals had no or little FDG nodal accumulation or any other hypermetabolic areas, whereas viremic individuals with early and advanced HIV had increased FDG in the peripheral nodes, indicating that FDG potentially identifies areas of HIV replication. FDG biodistribution was similar between early and advanced-stage disease. Four of 5 patients taken off HAART had negative baseline scans but developed nodal uptake and increases in viral loads. Furthermore, Brust et al and Liu showed that splenic uptake is significantly greater than liver uptake in the early infection group compared with HIV-negative or suppressed groups; similar findings are confirmed by our center (Fig. 1). This could reflect massive stimulation of $\mathrm{B}$ cells in the spleen by nonreplicating antigenic material. ${ }^{34,35}$

More recently, Lucignani et $\mathrm{al}^{36}$ assessed whether HIVinfected patients can be differentiated on the basis of the detection of specific locations of viral replication, even in the presence of an apparently optimal immunovirological response to HAART and whether FDG-PET findings can be related to immunovirological variables and AIDS history status. Patients under study were divided into 5 groups: subgroup Al (full responders, $n=8$ ): current HAART treatment, $\mathrm{CD}^{+} \mathrm{T}$ lymphocytes $>500 \mathrm{~mL}^{-1}$, and viral load $<50$ copies $/ \mathrm{mL}$; subgroup A2 (full responders, $n=5$ ): same criteria as A- 1 but with a history of AIDS; subgroup A3 (immunologic nonresponders, $n=5$ ): current HAART treatment, viral load $<50$ copies $/ \mathrm{mL}$, and low $\mathrm{CD}^{+} \mathrm{T}$ lymphocytes $\left(<200 \mathrm{~mL}^{-1}\right)$; group B (virological nonresponders, $\left.n=2\right)$ : current HAART treatment, $\mathrm{CD}^{+} \mathrm{T}$ lymphocytes around

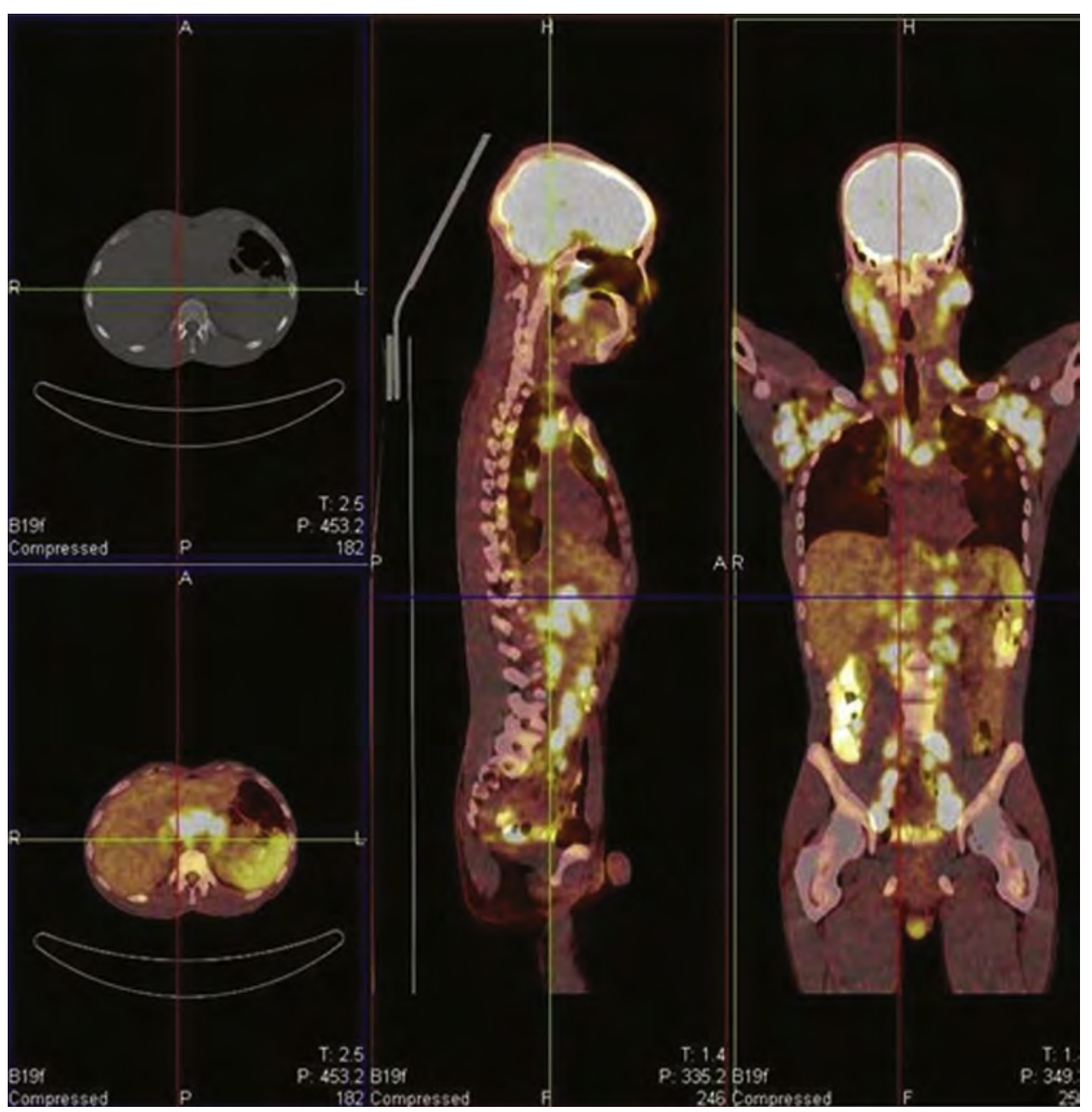

Figure 1 Diffuse FDG-avid lymphadenopathy in the cervical, axillary, hilar, abdominal, and pelvic nodes. With intense splenic uptake (ie, more than the liver). On a 28-year-old HIV-infected patient with lymphadenopathy. This demonstrate that HIV-1 infection progresses in distinct anatomical steps, with distinct lymphoid tissue activation in the head and neck during acute disease, a generalized pattern of peripheral lymph node activation at the midstages, and involvement of abdominal lymph nodes during late disease, suggesting that lymphoid tissues are engaged in a predictable sequence. 
$500 \mathrm{~mL}^{-1}$, and viral load $>50,000$ copies/mL; and group C (HAART naïve, $n=5$ ): no current or previous HAART treatment and increased viral load. PET images revealed different patterns of FDG uptake. All HAART-treated patients with either suppressed ( $<50$ copies/mL; group A) or high viremia (group B) showed a normal pattern of FDG uptake. On the contrary, the HAART naive subjects with high viremia (group C) displayed multiple foci of increased glucose metabolism in the LNs. In the HAART-naive subjects, FDG uptake, apparently related to viremia level, was observed in the upper torso mainly in the axillary nodes bilaterally in patients with viremia below 100,000 copies/mL; in those with viremia higher than 100,000 copies/mL, FDG uptake was also observed in the inguinal LNs. ${ }^{36}$

FDG-PET constitutes an in vivo, noninvasive imaging technique for disease state and its relation with immunovirological status indicates that it can be considered a marker of disease status.

\section{Fever of Unknown Origin (FUO)}

Classical FUO is discussed in details in this series. HIVassociated FUO can be defined as "fever $38.3^{\circ} \mathrm{C}$ or higher on several occasions, in a patient with a confirmed positive serology for HIV, with a duration of fever of 4 weeks or more for outpatients, or 3 days or more for hospitalized patients, with an uncertain diagnosis after 3 days despite appropriate investigation, including at least 2 days' incubation of microbiological cultures."37,38

Patients with HIV have an increased risk of fever owing to multiple factors. Infection with HIV itself can cause fever, ${ }^{39}$ as is the case in $40 \%-90 \%$ of patients with HIV primoinfection. ${ }^{40}$ Immunodeficiency in advanced HIV infection puts patients at high risk for opportunistic infections and malignancies. Drug treatment for HIV infection and its complications is a wellknown cause of febrile drug reactions. ${ }^{41}$ Sexually transmitted diseases, alcohol abuse, and illicit drug use are more frequent in patients with HIV than in the general population and may cause fever. $^{42}$

As per deliberations in the previous chapter, strategies for diagnosis and treatment of febrile episodes in patients with HIV should always involve initial clinical and first diagnostic investigations. If no diagnosis can be made after these first diagnostic investigations, PET/CT may be indicated. ${ }^{43,44}$ In patients with HIV and FUO, FDG-PET/ CT has emerged as a valuable tool for diagnosis, especially when CT anatomical landmarks are added to PET findings. ${ }^{45}$ The usefulness of FDG-PET/CT in FUO in HIVpositive patients was also validated by Martin et al, and their findings suggest that HIV viremia does not affect FDG-PET/CT performance. ${ }^{46}$

\section{AIDS-Associated Malignancy}

AIDS-defining cancers (ADCs), such as Kaposi sarcoma (KS), non-Hodgkin's lymphoma (NHL), and invasive cervical cancer, have significantly declined. On the contrary, non-AIDSdefining cancers (NADCs), such as Hodgkin's lymphoma (HL), anal cancer, hepatocellular carcinoma, lung cancer, and colorectal cancer, have gradually emerged as a major fraction of the overall cancer burden. ${ }^{47,48}$
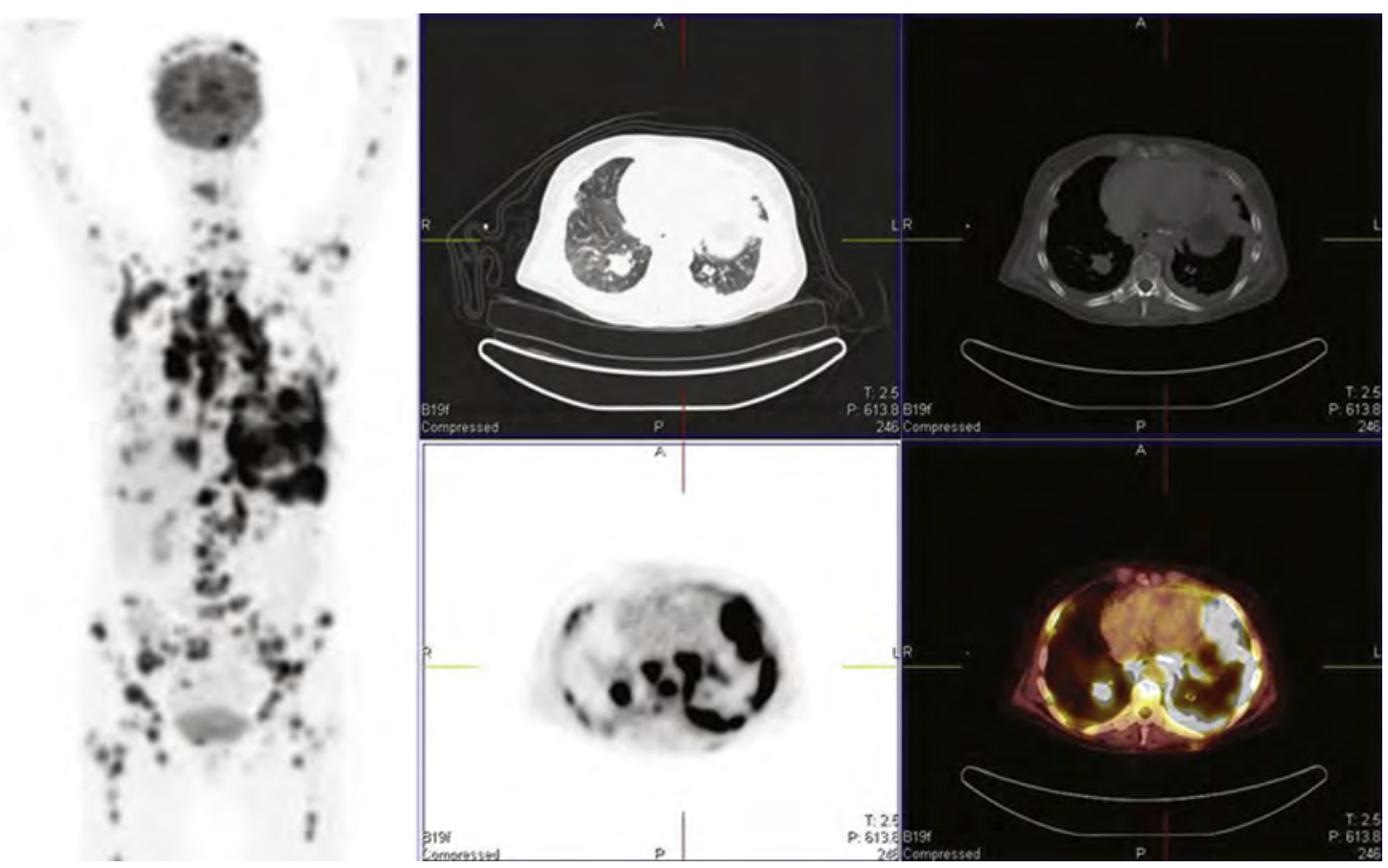

Figure 2 Intense FDG accumulation on both lungs and mediastinal and hilar lymph nodes, and diffusely increased FDG accumulation in bone consistent with widespread metastastatic disease. This illustrates that HIV-positive patients with lung cancer present with advanced disease (stage IV) presenting in younger age group (32 years) with lower CD4 count $(\mathrm{CD} 4=69)$. 
In a recent large prospective study, it was found that the latest CD4 cell count is strongly associated with the risk of death from both ADC and NADC. ${ }^{48}$ This finding has been confirmed by a recently published meta-analysis on the incidence of cancers in HIV/AIDS and transplant recipients, which revealed the importance of immunosuppression. ${ }^{49}$ Individual ADCs and related FDG-PET findings are discussed later.

NADCs are a very heterogeneous group of cancers with increasing importance in subjects with HIV infection. They develop in patients that are younger than general population and their clinical manifestations are usually atypical, with higher tumor grades, more aggressive clinical behavior and metastatic disease. ${ }^{50}$ The outcome is poor, with rapid progression, high rate of relapse, and poor response to treatment (Fig. 2). There are several factors that influence their development: HIV infection, chronic immunosuppression, and coinfection with some oncogenic viruses. The most frequent NADCs are those associated with human papillomavirus (HPV) infection (anal cancer), lung cancer, hepatic cancer, and HL. ${ }^{51,52}$ Their management is based on 3 essential points: the treatment of the specific malignancy, the use of ART, and the prophylaxis and treatment of opportunistic infections. The 2 factors significantly associated with prevention of NADCs are a $\mathrm{CD}^{+}$lymphocyte count more than 500/ $\mathrm{mm}^{52,53}$ and an undetectable viral load. The role of FDGPET/CT in these NADCs is similar to that in non-HIV-infected patients (Fig. 3).

\section{Cervical Cancer}

Cervical cancer remains the commonest malignancy in developing countries. The incidence of HIV infection amongst patients with cervical cancer is approximately $20 \%-25 \% .{ }^{54}$ In addition, patients present $10-15$ years earlier and with more advanced-stage disease. Immunosuppression influences patient performance status, tumor burden, tumor aggressiveness, tolerance to therapy, and outcome. ${ }^{55}$

HIV infection and low $\mathrm{CD}^{+}{ }^{+}$T-cell count are associated with an increased risk of persistent oncogenic HPV infection, the major risk factor for cervical cancer. Trends in HIVassociated cervical cancer have changed in the HAART era. Recent molecular pathways suggest that the natural progression of HPV infection, the causal agent in all cervical cancers, may be related to immune system dysfunction as well as HIV or HPV synergistic mechanisms. When highly active retroviral therapies are used, invasive cervical cancer treatments are affected by concomitant drug toxicities that could potentially limit therapeutic benefit of either HAART or, the standard of care treatment for locally advanced cervical cancer, concomitant chemoradiotherapy.

The assessment of response to radiotherapy in cervical has been hampered by the lack of suitable investigations with which to assess response. ${ }^{56}$ The role of FDG-PET/CT in cervical cancer has been well established. FDG-PET/CT demonstrates abnormal cervical uptake in virtually all patients with cervical cancer, and the current state-of-the-art PET
A

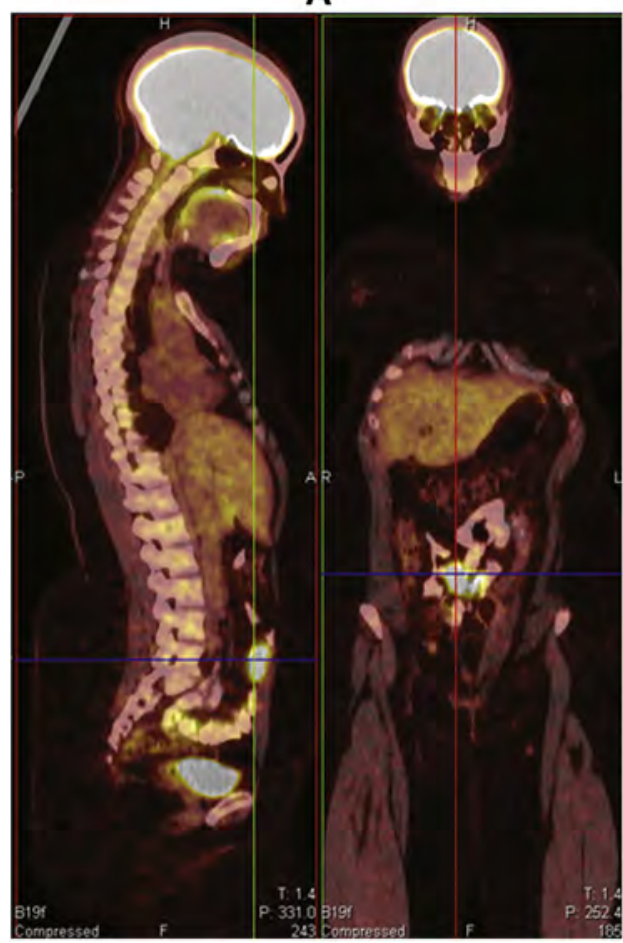

B

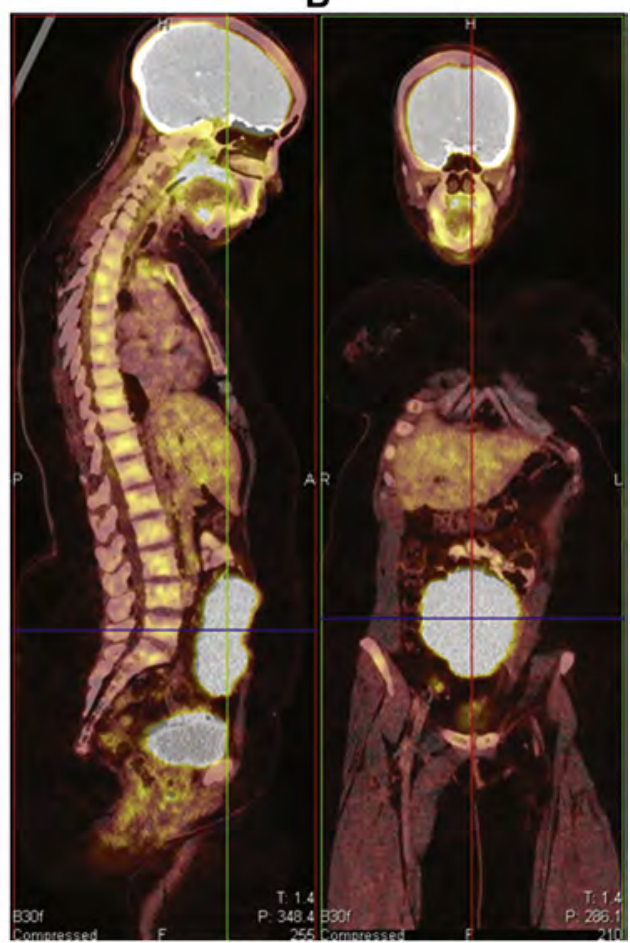

Figure 3 HIV-positive patients with cervical cancer demonstrate more rapidly progressive disease and have higher recurrence rates. In this young lady, FDG study revealed a pathologic uptake located in the umbilicus associated to peritoneal carcinomatosis. Consistent with Sister Mary Joseph nodule (A). Follow-up scan after 6 months demonstrated rapidly progressive disease (B). 


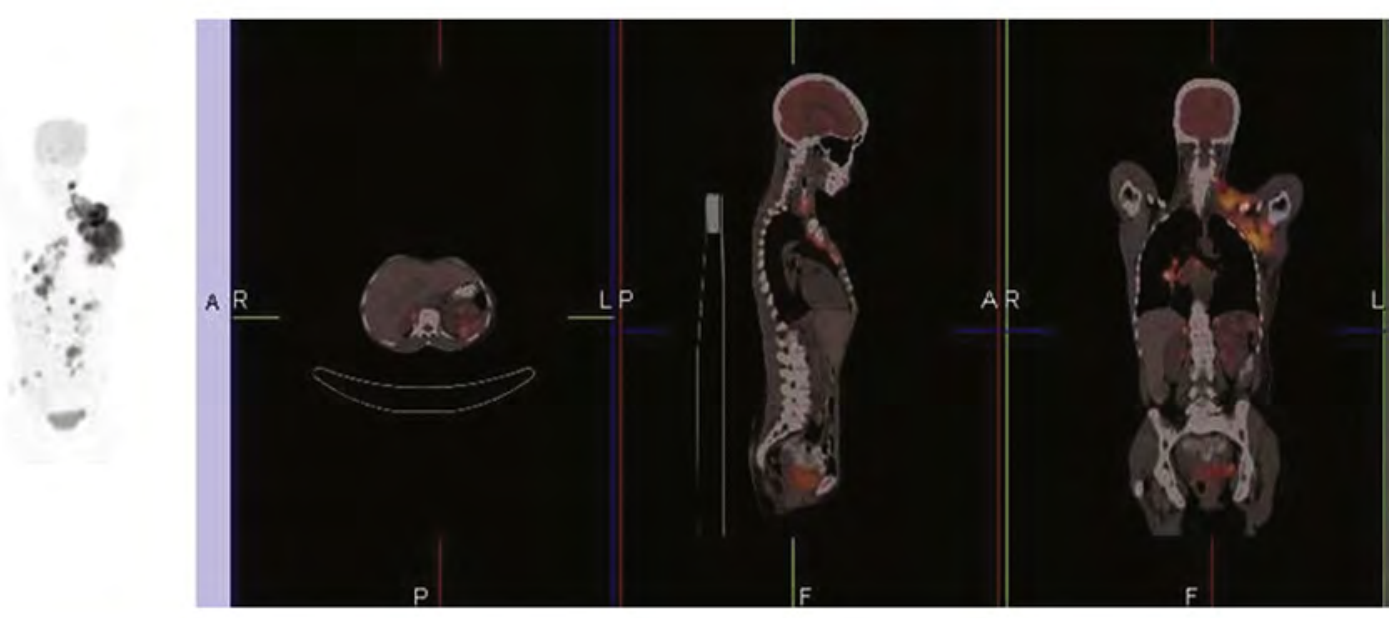

B

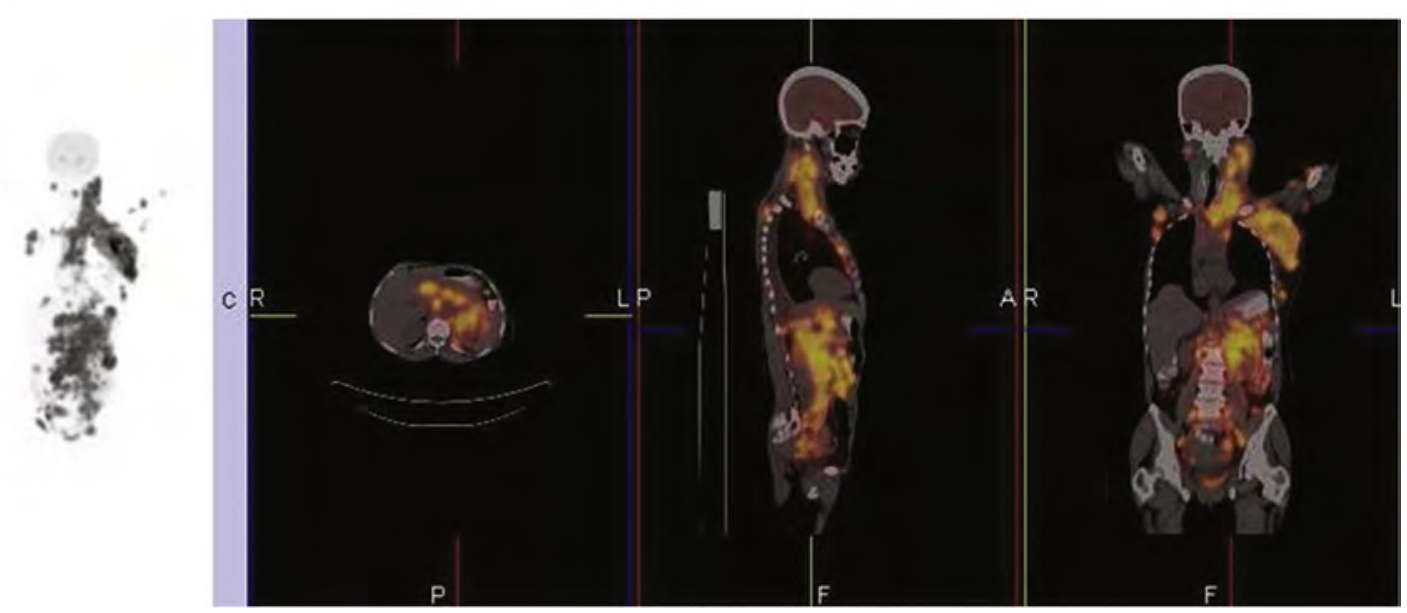

Figure 4 HIV-positive patient with DLBCL for therapy response or monitoring (after 8 courses of CHOP). Her CD4 = 354, viral load $<50$ (on antiretroviral medication for 17 months). FDG-avid lymphadenopathy in the left axilla region, hilar, para-aortic, abdominal, and pelvic nodes (A). The follow-up scan is more intense with involvement of the pelvic bones and shows rapidly progressive disease (B).

software allows for the 3-dimensional volumetric analysis of the tumor, which has been shown to be of more prognostic significance than clinical stage of disease. ${ }^{55,57}$ In a recent study by Kidd et al, pretreatment FDG-PET LN status, cervical tumor standardized uptake value (SUV) $\max$, and tumor volume combined in a nomogram proved a good model for predicting cervical cancer recurrence free survival, decision support system, and overall survival. This analysis is used in our center for prognostication of patients with HIV-positive cervical cancer. $^{58}$ And our ongoing experience demonstrates that FDG-PET/CT is a valuable technique for assessing prognosis, modifications to the chemoradiotherapeutic regimen, treatment response after the completion of concurrent chemoradiation, and suspicious or documented recurrence (Fig. 4).

\section{Kaposi Sarcoma}

KS was one of the earliest manifestations of the AIDS epidemic and the severe morbidity and mortality associated with AIDSKS prompted a vigorous research effort into its etiology which led eventually to the discovery of KS-associated herpesvirus or human herpes virus-8 (HHV8). ${ }^{6}$ Infections with HHV8 have been detected in all forms of KS and deemed the causative agent of both AIDS-defining and non-AIDS-related KS. ${ }^{59}$ Infections with HHV and HIV cause the activation of numerous preexistent and virus-specific signal transduction pathways. ${ }^{60}$ The introduction of effective ARTs in the mid-1990s led to a sharp decrease in the incidence of KS in HIV-infected subjects in developed countries where such therapies were widely available. ${ }^{61}$ A similar trend has been noticed in developing countries.

FDG-PET/CT was shown to be effective in detecting clinically occult KS lesions that were difficult to diagnose with traditional imaging techniques in more advanced stages of $\mathrm{KS}^{62,63}$ Visceral involvement predicts survival in patients with AIDS-associated KS, ${ }^{64}$ thus accurate staging and identification of more sites with FDG-PET/CT is useful in the management of this patients.

In addition to KS, KS-associated herpesvirus or HHV8 infection is associated with the plasma cell variant of multicentric Castleman disease (MCD), a rare lymphoproliferative 
disorder. In HIV-infected patients, MCD is highly aggressive and often lethal. ${ }^{65}$ Recently, Barker et al ${ }^{66}$ evaluated the efficacy of FDG-PET/CT in the diagnosis and monitoring of MCD activity. Nine patients with histologically confirmed MCD underwent fused FDG-PET/CT at initial diagnosis, after relapse, or during remission. ${ }^{66}$ FDG-PET/CT was able to detect abnormal uptake in LNs more frequently than CT-alonedetected enlargement. ${ }^{66}$ Scanning with FDG-PET/CT may therefore be useful in correctly identifying appropriate sites for biopsy, staging, and monitoring lymphoproliferation owing to HIV-associated MCD.

\section{Lymphoma}

The Incidence of aggressive B-cell lymphoma is dramatically increased in the HIV-seropositive population. ${ }^{67}$ Before the introduction of HAART, high-grade B-cell immunoblastic lymphomas (BCLs) were the most common histologic subtype followed by Burkitt lymphoma (BL) and intermediate-grade diffuse large BCL (DLBCL). ${ }^{67,68}$ Data since the introduction of HAART reveal a marked decrease in the incidence of immunoblastic lymphomas with an increase in DLBCL and BL. ${ }^{68}$

Higher viral load and lower CD4 counts are both risk factors for the development of NHL. ${ }^{69,70}$ The risk of NHL rises substantially in patients with HIV ribonucleic acid levels greater than 100,000 copies $/ \mathrm{mL}$ and those with CD4 counts of less than $50 \mathrm{~mL}^{-1}{ }^{69,71}$ Systemic lymphomas, however, may occur at virtually any level of CD4 count, with BL occurring more frequently in those with CD4 counts greater than $200 \mathrm{~mL}^{-1},{ }^{72,73}$ and primary central nervous system (CNS) lymphomas are associated with the most severe immunocompromise being seen almost exclusively in those individuals with CD4 counts less than $50 \mathrm{~mL}^{-1}$. ${ }^{74}$

$\mathrm{HL}$ is a non-AIDS-defining malignancy that has been shown to be increased in incidence in the setting of HIV infection, with large cohort studies and AIDS-cancer match registry data demonstrating standardized incidence ratios of approximately $9-18 .{ }^{67,75}$ In most series of cases, the histologic pattern differs from the general population in that mixed cellularity is the most commonly reported pattern rather than nodular sclerosis. Lymphocyte-depleted histology has also been observed more often in the setting of HIV infection.

${ }^{18} \mathrm{~F}$-FDG-PET/CT is a valuable imaging technique in the diagnosis, staging, restaging, and monitoring response to therapy in HIV-infected patients with lymphoma (Fig. 4). ${ }^{76}$ The most common subtypes of NHL diagnosed in HIVinfected individuals are DLBCL and BL. These lymphomas are usually high grade and often widely disseminated with frequent involvement of extranodal sites at the time of diagnosis. $^{76}$

As described before, patients with HIV-1 are at risk for lymphoma, making the differential diagnosis between benign and malignant lymphadenopathy imperative. Goshen et $\mathrm{al}^{77}$ studied 7 patients with HIV-1. Six patients with known NHL underwent a total of 16 PET/CT scans; 5 of 16 scans were performed for initial staging and 10 of 16 for evaluating treatment response and follow-up. One patient was referred for evaluation of lymphadenopathy suspected of being lymphoma. PET/CT findings were compared with concurrent clinical, immunologic, and virological data. PET/CT accurately depicted the extent of lymphoma in 12 of 16 patients' scans (75\%), yet in 4 of 16 (25\%) scans increased FDG uptake was noted in LNs of normal CT appearance (PET+/CT-). The highest values of CD4 cell count and viral load were concurrent with the discrepant PET+/CT - scans, seen in 4 scans, in 2 patients. The PET+/CT - findings in both these patients were observed in neck, axillae, mediastinum, spleen, and inguinal regions, and sample biopsies of the PET $(+)$ nodes consequently proved benign findings in both patients. The specificity of FDG uptake in LNs in patients with HIV is not only problematic in ADCs but also in NADCs. ${ }^{78}$ Finally, Just et $\mathrm{al}^{79}$ retrospectively studied 13 consecutive HIV-positive patients with BL who underwent 1 or more PET/CT scans. In 5 of 5 patients imaged before treatment, PET/CT confirmed all involved sites detected at conventional workup and demonstrated additional sites in 4 of 5 patients. LN involvement, which is known to be uncommon in endemic or sporadic BL, was present in 54\% of patients. Additionally, in 3 patients, BL was predominantly located in parotid LNs, which is also an unusual finding. A negative scan was encountered in 3 of 10 patients imaged during treatment and in 1 of 4 patients imaged after treatment completion and was always associated with lasting complete remission. FDGPET/CT may help guide treatment strategy and minimize longterm toxicity in lymphoma patients with HIV-1 and had been used confidently as a management tool in this patient group. ${ }^{80}$ As suggested by Goshen et al, in the context of discrepant PET/ CT findings, increased viral loads and CD4 levels may imply benign HIV-related lymphadenopathy.

\section{FDG-PET to Differentiate Opportunistic Infections from Lymphoma in the Brain}

The distinction between primary CNS lymphoma and nonmalignant lesions due to opportunistic infections, in particular cerebral toxoplasmosis, is important as treatment is different. Cerebral toxoplasmosis can be effectively treated with medication, whereas primary CNS lymphomas are treated with radiation therapy and corticosteroids. Neither CT nor MRI can reliably distinguish CNS infections, such as toxoplasmosis, from lymphoma in HIV-1-positive patients. Accordingly, the potential of FDG-PET to noninvasively differentiate cerebral toxoplasmosis and other infectious diseases from primary CNS lymphoma has been assessed by several authors. Hoffman et $\mathrm{al}^{81}$ studied 11 individuals with AIDS and CNS lesions with FDG-PET. Both qualitative visual inspection of the PET images as well as semiquantitative analysis indicated that FDG-PET was able to accurately differentiate between a lymphoma and a nonmalignant etiology for the CNS lesions. Similar results were found by Villringer et al. ${ }^{82}$ These authors examined 11 patients with AIDS, 6 with toxoplasmosis, 1 with a tuberculoma, and 
4 with a primary CNS lymphoma. The FDG uptake within the lesion was compared with the uptake in a contralateral brain area (SUV). In all subjects with cerebral infections (toxoplasmosis and tuberculoma), the SUV ratio was significantly lower than the SUV ratio in patients with lymphoma with no overlap of the uptake values. Heald et $\mathrm{al}^{83}$ studied 18 patients that were divided into 3 groups based on biopsy results, serology, and response to therapy. Group 1 consisted of 8 patients with infectious lesions (4 with toxoplasmosis, 2 with neurosyphilis, and 2 with progressive multifocal leukoencephalopathy). Group 2 consisted of 5 patients with biopsy-proven CNS lymphoma. Group 3 consisted of 5 patients with presumed CNS lymphoma. Using both a qualitative visual score and a semiquantitative count ratio comparing the lesion with contralateral brain, CNS lesions diagnosed as lymphomas had statistically higher visual scores and count ratios than CNS lesions diagnosed as infections.

Finally, O'Doherty et al ${ }^{84}$ showed that in 19 patients who were on antitoxoplasmosis treatment, FDG-PET scan was $100 \%$ sensitive and specific for identifying primary CNS lymphoma. Hence, FDG-PET is valuable in atypical CNS lesion detection, not only to differentiate the primary CNS lymphoma from infections but also to identify the optimal site for biopsy, and thus reduce the risk of complications by demonstrating the easiest accessible brain lesion.

\section{PET Imaging With FDG in ADC}

HAND, formerly known as ADC, is a subcortical dementia that is characterized by disturbances in cognition, motor performance, and behavior. Diagnosis of early HAND is important as many of its symptoms can be caused by other conditions common to people with HIV or AIDS, many of which may be treatable. ${ }^{85}$ There is no diagnostic marker or combination of markers for HAND. The diagnosis is made in HIV-positive patients with cognitive impairment after ruling out confounding conditions (CNS opportunistic infections, neurosyphilis, substance abuse, delirium, toxic-metabolic disorders, psychiatric disease, and age-related dementias). An essential feature in the diagnosis of HAND is the presence of well-documented cognitive decline and the exclusion of other neurologic complications of HIV infection, such as cerebral toxoplasmosis, cryptococcal meningitis, lymphoma, and progressive multifocal leukoencephalopathy, conditions that are characterized by focal or heterogeneous ${ }^{18} \mathrm{~F}-\mathrm{FDG}$ uptake as opposed to the striatal symmetrical hypermetabolism observed in early HAND. ${ }^{86}$ Therefore, cerebrospinal fluid examination and imaging studies of the brain are mandatory. Cerebrospinal fluid analysis should exclude infectious agents other than HIV.

Although the primary imaging methods to enhance diagnosis of neurologic complications associated with HIV infection are MRI and CT, functional imaging may prove to be of greater value because HAND/ADC causes functional abnormalities before structural atrophy, ventricular dilatation, or focal CNS lesions are visible. ${ }^{87} \mathrm{~A}$ number of studies have reported on FDG-PET brain findings in demented AIDS patients as well as in asymptomatic HIV-infected subjects. ${ }^{79-93}$ In an early imaging study using FDG-PET in 12 patients with dementia, Rottenberg et $\mathrm{al}^{94}$ found relative subcortical hypermetabolism in 9 patients in the early stage of AIDS-related dementia. Disease progression was characterized by reduced glucose uptake in cortical and subcortical gray matter (Fig 7). In a follow-up study by the same group, 21 HIV-infected subjects (11 with AIDS) were examined. Twelve had follow-up scans at 6 months and 4 had a third scan at 12 months. Principal component analysis of the combined (HIV-infected and controls) PET data revealed 2 major disease-related metabolic components: a nospecific indicator of cerebral dysfunction, which was significantly correlated with age, cerebral atrophy, and HAND or ADC stage; and the striatum, which was hypermetabolic and appeared to provide a diseasespecific measure of early CNS involvement. ${ }^{91}$ Similar findings were reported by Van Gorp et al who described regional hypermetabolism in the basal ganglia and the thalamus in 17 subjects with AIDS when compared with 14 seronegative controls. ${ }^{95}$ The authors also found a significant relationship between temporal lobe metabolism and the severity of dementia. Hinkin et a ${ }^{89}$ showed that as HIV-associated brain infection progressed, relative basal ganglia metabolism increased as well as metabolism in the parietal lobe. Pascal et $\mathrm{a}^{95}$ found in 10 of 15 asymptomatic HIV-positive patients, significant asymmetries in FDG uptake which were most prominent in the frontal regions, whereas MR showed no abnormalities. Finally, during a motor task, von Giesen et al ${ }^{93}$ found frontomesial hypometabolism in 9 of 19 nondemented HIV-infected subjects. The authors indicated that frontomesial hypometabolism was associated with deteriorating motor performance.

\section{Cardiovascular Disease (CVD) and Arterial Inflammation}

People living with HIV have a 2-fold greater risk for experiencing a stroke or myocardial infarction than the general population. ${ }^{96}$ Evidence suggests that chronic low-grade inflammation associated with the host immune response to HIV infection and ongoing viral replication contributes to greater CVD risk and the higher incidence of CV events in HIVinfected adults. ${ }^{97,98}$

Several groups have pioneered the use of ${ }^{18} \mathrm{~F}$ FDG uptake by proinflammatory macrophages present in the arterial wall as a noninvasive, sensitive, specific, and reproducible molecular level biomarker for early atheroma formation in metabolically active, rupture-prone atherosclerotic plaques. ${ }^{99-101}$ Proinflammatory macrophages utilize glucose at a high rate. ${ }^{100}$ Similarly carotid ${ }^{18}$-FDG-PET/CT imaging detected significant vascular inflammation in HIV-infected men and women with low Framingham coronary heart disease risk scores, suggesting that this molecular imaging method is sensitive to early proatherosclerotic processes in a clinical population suspected of having chronic, low-grade inflammation-induced CVD. ${ }^{102}$ This was confirmed by 
Subramanian et al. ${ }^{103}$ Currently there is a paucity of knowledge and more research is needed to examine the potential role of PET in diagnosis of HIV and CVD.

\section{FDG-PET Findings in HIV-1- Infected Patients With Lipodystrophy}

A chronic and progressive syndrome of lipodystrophy (peripheral fat loss or abdominal obesity or both) and hyperlipidemia has been reported in up to $60 \%-80 \%$ of patients treated with HAART. ${ }^{104,105}$ Hyperinsulinemia, increased C-peptide concentration, insulin resistance, and impaired glucose tolerance have been frequently observed. Insulin resistance, as determined by euglycemic-hyperinsulinemic clamp technique, reflects defective insulin action observed predominantly in skeletal muscle and liver. Behrens et al ${ }^{106}$ determined wholebody glucose disposal and oxidation by combination of the euglycemic-hyperinsulinemic clamp technique and indirect calorimetry in patients receiving HAART with signs of lipodystrophy as confirmed by dual energy x-ray absorptiometry. Muscle glucose uptake of the thighs was measured simultaneously by dynamic 2 FDG-PET. Whole-body glucose disposal was significantly reduced in these patients compared with untreated patients. Analysis of kinetic constants using a 3-compartment model indicated reduced skeletal glucose uptake caused by significantly impaired glucose transport and phosphorylation. Skeletal muscle glucose uptake was reduced by $66 \%$ in treated patients and explained $46 \%$ and $43 \%$ of whole-body glucose disposal in patients on HAART and therapy-naive patients, respectively. Insulin-stimulated whole-body oxidative and nonoxidative glucose disposal was significantly lower in the treated group, as was suppressive insulin action on lipolysis. Bleeker-Rovers et $\mathrm{al}^{107}$ performed FDG-PET in 4 HIV-infected patients with early features of lipodystrophy treated by a stavudine-containing HAART regimen for a maximum of 3 years, in 4 HIV-infected patients never treated by HAART (control 1), and in 5 HIV-infected patients without lipodystrophy treated for a maximum of 3 years by HAART that never included stavudine; stavudine-based regimens have the highest incidence of lipoatrophy and switching treatment to zidovudine or abacavir may reduce lipodystrophy. In lipodystrophy patients with markedly increased subcutaneous ${ }^{18}$ F-FDG uptake, stavudine was replaced by zidovudine, abacavir, or tenofovir and FDG-PET was repeated 3-6 months later. Markedly increased subcutaneous ${ }^{18} \mathrm{~F}$-FDG uptake was found in 3 of 4 HIV-infected patients with lipodystrophy but in none of HIV-infected controls without lipodystrophy. Likewise, in our series of 39 patients suffering from HIV, 7 drug-naive patients, 21 nonlipodystrophic patients on HAART, and 11 patients on HAART suffering from lipodystrophy, quantitatively, FDG uptake by subcutaneous fat proved significantly higher in HIV patients under HAART presenting with lipodystrophy (Fig. 5) ${ }^{108}$ Without the knowledge of this
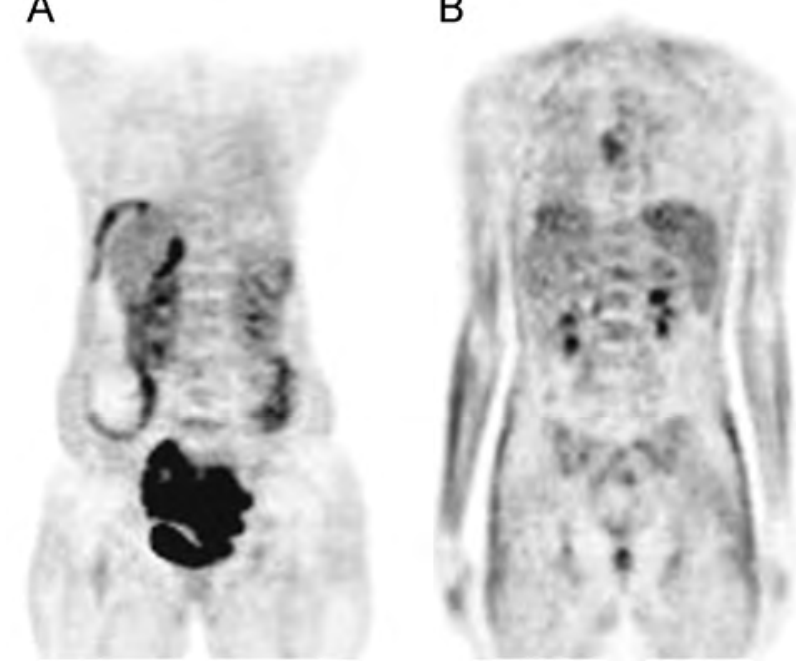

Figure 5 A coronal image of a patient under highly active antiretroviral therapy (HAART) showing normal FDG uptake in subcutaneous fat (A). A coronal image of a patient under HAART with lipodystrophy with increased FDG uptake in subcutaneous fat (B). The patient with lipodystrophy had the clinical evidence from physicians' and patient's reports of fat wasting from the face, buttocks, limbs, and upper trunk, and accumulation of fat in the abdomen or over the dorsocervical spine, and no history of anabolic steroids, glucocorticoids, or immunomodulators within 3 months of assessment.

phenomenon, this uptake pattern may be misinterpreted as diffuse soft tissue inflammation, or even as a technical fault with attenuation correction. The potential role of FDG-PET in monitoring response to various treatment options aimed at reducing insulin resistance in lipodystrophic HIV patients under HAART warrants further investigation. ${ }^{109}$ As this may help in modification of newer HAART and regimens.

\section{Thymic FDG-PET Findings After HAART}

The thymus in adults infected with HIV-1 is generally thought to be inactive, both because of age-related involution and viral destruction. ${ }^{110}$ The observed increase in the number of $\mathrm{CD} 4 \mathrm{~T}$ cells after HAART has been attributed to thymic reactivation by some authors. However, this issue remains controversial. Hardy et $\mathrm{al}^{111}$ performed sequential FDG-PET imaging in patients under HAART therapy. In 1 patient, clear correlations could be drawn between visible activity within the thymus, as measured by increased FDG uptake, and regeneration of naive CD4 (CD45RA/CD62L) T cells, increased numbers of CD4 T cells, controlled viremia, and increased numbers of recent thymic emigrants. A second patient displayed no increase in peripheral CD4 count and no increase in thymic activity. Similar findings were confirmed by Tanaskovic et $\mathrm{al}^{112}$ who found no residual thymic tissue on FDG-PET in some previously immunodeficient HIV patients responding to ART that display poor recovery of $\mathrm{CD} 4^{+} \mathrm{T}$ cells.

Recently, Lelièvre et al have indicated that FDG-PET/ $\mathrm{CT}$ in HIV may serve as a new marker for the evaluation 
of thymic function in HIV-infected patients. Their study also showed that thymic function is increased in HAART-naive HIV patients and relatively impaired in older chronically treated patients arguing for a place in the treatment of HIV infection of strategies aimed to restore thymic function. Without knowledge of thymic reactivation in adults, these studies may be falsely reported as being positive for disease in the anterior mediastinum.

\section{Tuberculosis}

In HIV/AIDS and TB infection, early identification and diagnosis of active TB is the key to effective control of the disease. ${ }^{113}$ Globally, TB continues to be one of the leading infectious causes of morbidity and mortality, accounting for an estimated 9 million cases and 2 million deaths per year. ${ }^{114,115}$

The effect of the dual HIV and TB epidemics is felt most severely in developing countries, with recent estimates of dual infection rising rapidly. HIV-infected patients are at a 20-fold increased risk of contracting TB when compared with HIVnoninfected peers. ${ }^{114,116}$ Therefore, there is an increasing clinical need for effective imaging for the following:

(1) Early identification and diagnosis of TB (key to effective control of the disease);

(2) Differentiation between malignancies and TB, which is particularly important for solitary pulmonary nodules

(3) Correct identification of extrapulmonary TB and staging of TB (crucially important to start anti-TB treatment and coordinate this with ART for HIV);

(4) Follow-up of TB patients (in developing countries, multidrug resistant and extensively drug resistant TB are of serious consequence, and thus monitoring of therapy is essential).

\section{Pulmonary}

Diagnosing active pulmonary TB is one of the major challenges in the clinical management of active pulmonary TB. The conventional diagnostic strategy is based on history, clinical signs and symptoms, microbiology (sputum smear and culture), and chest x-ray (CXR). The classical radiographic findings of pulmonary $\mathrm{TB}$, consisting of upper lobe involvement and cavitations without lymphadenopathy are usually only found in HIV-infected individuals without severe immunosuppression (CD4 > 200). In those HIV-infected individuals with severe immunsuppression (CD4 < 200), atypical radiographic pattern is predominant (Mendelson ${ }^{117}$ ). This includes involvement of the middle and lower parts of the lungs, the lack of cavity formation, presence of lymphadenopathy, pleural effusion, and military pattern. Nevertheless, a number of patients may even present with a normal CXR. The radiographic appearance can be confused with copathologies of the lung frequently seen in HIV-infected individuals, such as Pneumocystis jiroveci pneumonia or nontuberculous mycobacterium infections; bacterial, fungal, or viral pneumonia; KS; or NHL. Determination of pulmonary tuberculoma activity is mandatory for optimal treatment strategy. ${ }^{118}$ As FDG is known to accumulate in inflammatory cells such as neutrophils and activated macrophages at the site of inflammation, ${ }^{119,120}$ this enables FDG-PET to be the preferred modality for real-time assessment of disease activity over CXR, CT, and MRI. Goo et $\mathrm{al}^{121}$ studied 10 consecutive patients suffering from histopathologically proved pulmonary TB. Nine of 10 tuberculomas showed FDG uptake at PET, and the mean $\mathrm{SUV}_{\max }$ value was $4.2 \pm 2.2 \mathrm{SD}$. FDG uptake (range 1.9-3.7) in lesions adjacent to main abnormalities was demonstrated in 4 patients. The authors concluded that pulmonary tuberculoma commonly causes an increase in FDG uptake.Recently, Kim et al evaluated the potential role of double-phase acquisition of FDG-PET for the differentiation of active pulmonary tuberculoma. ${ }^{122}$ A total of 25 consecutive patients with pulmonary tuberculoma were enrolled. PET/CT imaging was performed 60 (range 53-71) and 120 minutes (range 109131) after injection of ${ }^{18}$ F-FDG. Early and delayed $S U V_{\max }$ values as well as \%Delta $\mathrm{SUV}_{\max }$ values were obtained in all patients. Active tuberculoma showed statistically significant higher values in maximal $\mathrm{SUV}_{\operatorname{maxE}}$ than those of inactive tuberculoma (active $=2.3 \pm 0.75$, inactive $=0.79 \pm 0.15$ ), $\mathrm{SUV}_{\operatorname{maxD}}$ (active $=2.48 \pm 0.79$, inactive $=0.75 \pm 0.13$ ), and \%Delta $\mathrm{SUV}_{\max }$ (active $=8.07 \% \pm 7.77 \%$, inactive $=$ $-3.83 \pm 6.59$ ). When $\mathrm{SUV}_{\operatorname{maxE}} 1.05$ was used as the cutoff point, the sensitivity and specificity were $100 \%$ and $100 \%$, respectively. When $\mathrm{SUV}_{\operatorname{maxD}} 0.97$ was used as the cutoff value, the sensitivity and specificity were $92.8 \%$ and $100 \%$, respectively. When \%Delta $\mathrm{SUV}_{\max } 6.59$ was used as the cutoff value, the sensitivity and specificity were $71.4 \%$ and $100 \%$, respectively. The authors concluded that further studies are needed to confirm their results and improve statistical accuracy. In the series presented, 7 of 16 patients suffered from active lung TB. When compared with the series by Goo et $\mathrm{al}^{121}$ and Kim et $\mathrm{al}^{122}, \mathrm{SUV}_{\max }$ values in lung tuberculoma proved higher [mean values of 8.2 (range: 3.4-21.7) and 11.1 (range: 4.5-27.2), respectively]. ${ }^{120}$ Also, \%Delta SUV $\max$ proved higher in this series when compared with the findings by Kim et $\mathrm{al}^{122}$ (mean: 35, range: 23-54). From a clinical point of view, the high SUV values and the SUV increase over time observed are of concern when considering the specificity of cancer diagnosis in patients with solitary lung nodules. From a physiopathological point of view, the uptake of FDG in lung tuberculoma as found in our series and those by Goo et al and Kim et al likely relates to the nature of tuberculous granuloma. Granulomas are composed mainly of clusters of macrophages (ie, the hard granuloma). Blood vessels are usually scanty or completely absent in it and the tissue is hypoxic or anoxic. ${ }^{123-125}$ Macrophages adapt to hypoxia and survive on anaerobic glycolysis. ${ }^{126,127}$ As a consequence, the increased glycolysis of macrophages may result in a high SUV of FDG indistinguishable from malignancy.

In some patients, dual-phase ${ }^{18}$ F-FDG imaging may be useful to determine whether a site of pulmonary uptake of ${ }^{18}$ F-FDG is malignant or benign. The general basis of this theory is that although both pathologies may have an initially 
high $\mathrm{SUV}_{\text {max }}$, malignant tissues would have a greater retention of ${ }^{18} \mathrm{~F}-\mathrm{FDG}$ between 1 and 2 hours than benign processes. ${ }^{120,128}$ However, in these studies there was only 1 patient with TB in the noncancer group. The situation is very different when the benign disease group contains patients with TB. In a prospective series of 31 patients with suspicious pulmonary lesions, low washout (eg, a high-retention index for ${ }^{18}$ F-FDG) was seen in $60 \%$ of the benign lesions including 10 patients with TB. This was similar to a high-retention index of $62 \%$ at the malignant sites of disease; the inference being that late imaging and looking at washout rates or retention index of ${ }^{18}$ F-FDG is not useful. ${ }^{129}$ This study has been confirmed in a sub-Saharan population in a group of 30 patients with a high incidence of TB. The SUV $\max$ in the benign group was higher than those with cancer, ${ }^{130}$ which meant that the traditional $\mathrm{SUV}_{\max }$ cutoff of 2.5 was used and the specificity of ${ }^{18} \mathrm{~F}$-FDG was only $25 \%$ for finding cancer. Again, the washout of ${ }^{18} \mathrm{~F}$ FDG as measured by change in $\mathrm{SUV}_{\max }$ was almost identical in the 2 groups especially when TB was compared with cancer. It would, therefore, seem that the use of washout rates for ${ }^{18}$ F-FDG is unlikely to be helpful in differentiating TB from other causes of ${ }^{18} \mathrm{~F}$-FDG uptake in the TB endemic area such as sub-Saharan population (Fig. 6).

\section{Extrapulmonary}

Extrapulmonary TB (EPTB) refers to $\mathrm{TB}$ outside of the lungs. ${ }^{131}$ EPTB has been an AIDS-defining condition, indicating clinical stage 4 in adults. ${ }^{132,133}$

Although EPTB is seen in 15\%-20\% of immunocompetent patients with TB, EPTB is seen in more than 50\% of HIVpositive patients with TB; the risk of EPTB and of mycobacterial infection increases with advancing immunosuppression. ${ }^{134}$ The most common site of EPTB involvement is the LNs followed by pleural effusion and bone and joint involvement. ${ }^{134-136}$

EPTB presents a difficult diagnostic challenge for a nuclear physician or radiologist and requires a high index of suspicion, particularly in high-risk populations.

With regards to musculoskeletal TB, the spine is the most frequent location and usually the wide range of clinical presentations results in difficulties and delays in diagnosis (Fig. 7). To address this poor record, FDG-PET seems to be superior to both morphologic and conventional nuclear medicine imaging. PET demonstrate lesion extent, serve as guide for biopsy with aspiration for culture, assist surgery planning, and contribute to follow-up. ${ }^{137}$



Figure 6 PET/CT slides showing a pulmonary nodule in the left upper lobe which is ${ }^{18} \mathrm{~F}$-FDG avid on double-phase imaging. These features are indistinguishable for malignancy and TB; this patient was proven to have TB. 


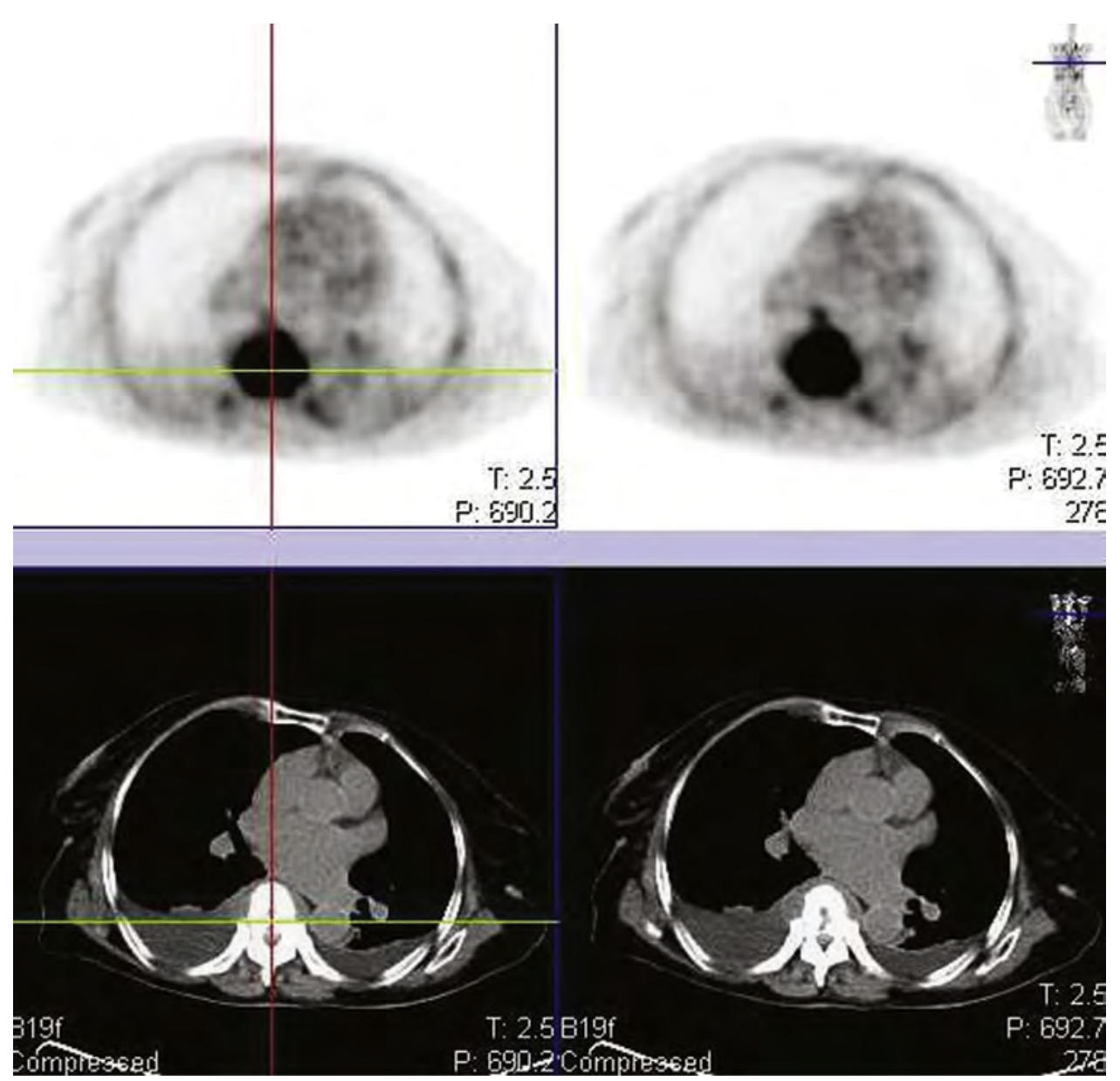

Figure 7 Consecutive transaxial PET/CT slides showing intense ${ }^{18}$ F-FDG in a patient with TB of the spine and low-grade ${ }^{18}$ F-FDG uptake in a pleural effusion. FDG-PET/CT provides more accurate assessment of the extent of involvement in patients suffering from extrapulmonary tuberculosis and thus a useful tool in the assessment of disease activity before treatment and determining the duration of treatment.

Sathekge et $\mathrm{al}^{138}$ studied the potential effect of dual-phase FDG-PET vs routine staging in $16 \mathrm{~TB}$ patients. In 9 patients, 18 sites of LN involvement were identified on both early and delayed images. Nine of 18 sites of LN involvement, occurring in 5 patients, were missed on CT. In 4 of these 5 patients, sites of LN involvement were the only sites of EPTB identified. Sites of osseous involvement and joint involvement, respectively, occurring in 4 and 3 patients, were identified on both CT and FDG-PET. Thus supporting that FDG-PET/CT can demonstrate lesion extent, serve as guide for biopsy with aspiration for culture, assist surgery planning, and contribute to follow-up.

\section{Therapy Monitoring of TB}

In developing countries, multidrug resistant and extensively drug resistant $\mathrm{TB}$ are of serious consequence, and thus monitoring of therapy is essential. A major attribute of PET stems from its ability to quantitate ${ }^{18}$ F-FDG uptake, which allows the infectious or inflammatory processes to be monitored during the early course of the disease. ${ }^{139}$ This is especially true in determining the effectiveness of therapy.
It is common for patients with low $\mathrm{CD}^{+}$counts and $\mathrm{TB}$ coinfection who are treated with ART to develop immune reconstitution inflammatory syndrome and frequently die because of this condition. Symptoms include new or worsening fever, lymphadenopathy, pulmonary, visceral, CNS, or cutaneous disease, which may be severe and life-threatening and must be differentiated from disease progression. Published evidence clearly demonstrates that TB-HIV integration is essential for improved survival, but the question of when to start ART during TB treatment is more complex. ${ }^{8}$ Fortunately, clinical trial evidence now demonstrates improved survival in severely immunocompromised TB-HIV coinfected patients with early HAART initiation from the start of TB treatment, except in patients with TB meningitis. ${ }^{8}$ Hence, early detection and monitoring of treatment is essential.

The treatment of TB can be problematic, the disease itself may be resistant to standard treatment, and treatment itself is prolonged with regimens in HIV-positive patients being anywhere from 3-9 months. Hospitalization for this period of time is not possible, so treatment is performed as an outpatient. However, this raises the problem of compliance, especially if the patient starts to feel better. The time course of treatment is empirical and tends to be the maximum needed to treat TB in 

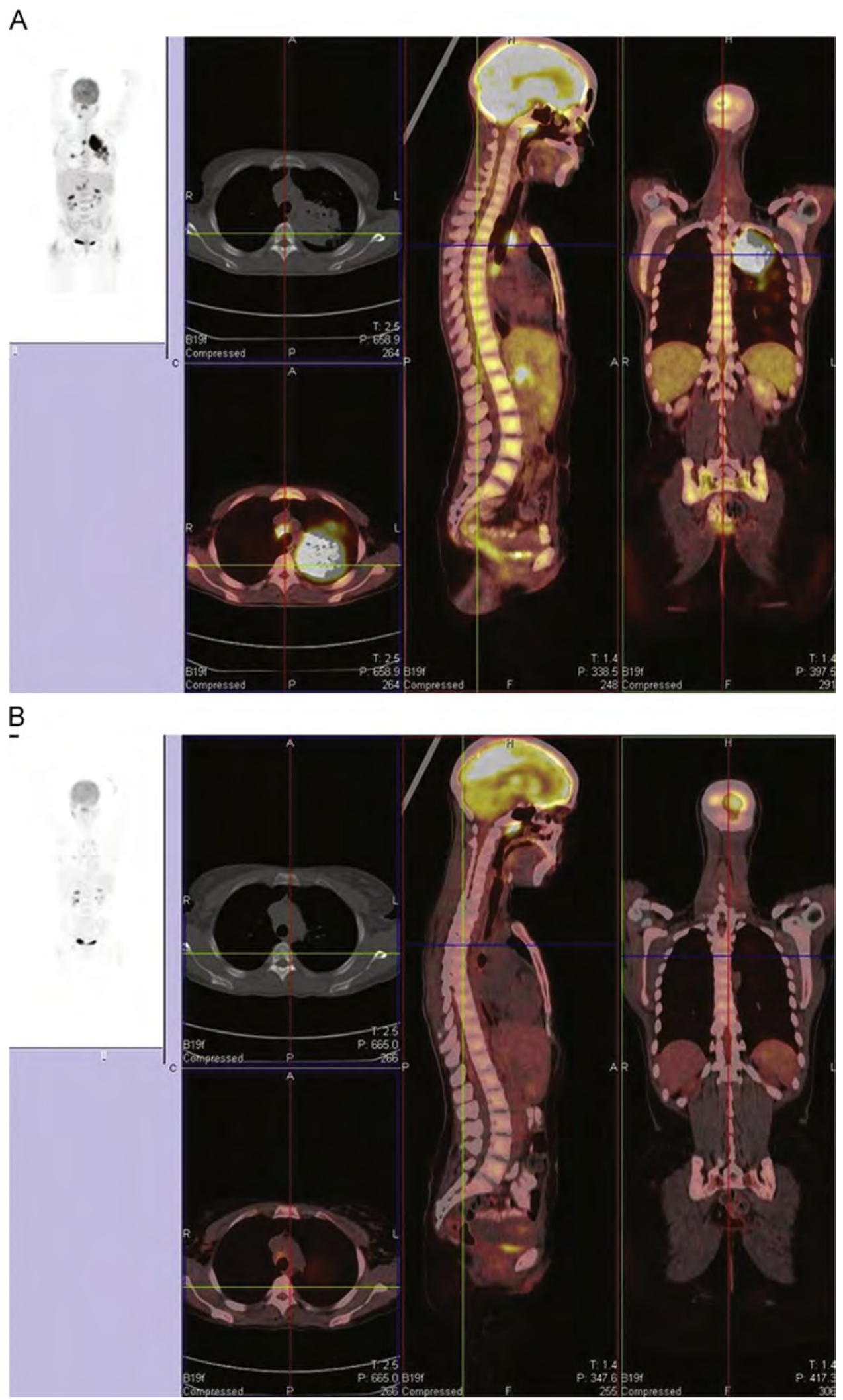

Figure 8 FDG-PET/CT performed before (A) and after therapy (B). The pathologic uptake in the left lung and mediastinum before therapy almost completely disappeared after therapy. This case highlights metabolic response may indicate clinical response and guide duration of antimicrobial therapy. 
that clinical situation. However, there can be great variation in the length of the course of treatment required, so an objective method to monitor treatment is required. Using an animal model and TB infection model, it has been shown that quantitative ${ }^{18}$ F-FDG uptake can be used to monitor the effectiveness of treatment. ${ }^{115,140}$ In this study, there was a clear correlation between the fall in ${ }^{18} \mathrm{~F}$-FDG uptake in the lungs and successful administration of a bactericidal drug. In contrast, uptake of ${ }^{18}$ F-FDG increased when an ineffective drug was given and in the control group. In humans, data by Demura et $\mathrm{al}^{141}$ indicate that ${ }^{18}$ F-FDG-PET could be an important noninvasive method for the diagnosis of latent and active disease that enables management of pulmonary mycobacterial infection as well as for assessment of therapeutic response in patients with pulmonary mycobacteriosis. This study, however, predominantly included patients suffering from nontuberculous pulmonary mycobacteriosis ${ }^{141}$ and confirmation of their findings in TB patients are needed. Park et $\mathrm{al}^{142}$ have demonstrated that during anti-TB treatment, some bacillusnegative tuberculomas do not decrease in size or may even keep growing, making it difficult for physicians to decide whether to alter treatment regimens. ${ }^{18} \mathrm{~F}-\mathrm{FDG}$-PET imaging may therefore be useful in this situation. If FDG-PET shows that SUV is increased, it is likely that the tuberculoma is in active status, and that treatment should be prolonged or the patient switched to other drugs. If the SUV is decreased, it is likely that the tuberculoma is responsive to anti-TB treatment, and current treatment should be continued according to standard protocols (Fig. 8). PET imaging may also be useful in differentiating between an inactive granuloma and a granuloma in the latent or active stage of infection. ${ }^{142}$ This was also proven in separate studies by Tian et $\mathrm{al}^{143}$ and Hofmeyr et al. ${ }^{144}$ Furthermore, patients with malignancy receiving immunosuppressive therapy are at risk for TB that may present as asymptomatic extrapulmonary disease. Although definitive diagnosis and exclusion of active TB infection by culture and examination of tissue specimens is always recommended, FDG-PET/CT may contribute to earlier diagnosis. ${ }^{144}$ In a study by our group, ${ }^{139} \mathrm{SUV}_{\max }$ of involved LN basins (both early and delayed) and number of involved LN basins on pretreatment scans proved significantly higher in nonresponders than in responders (respective $P$ values were $0.03,0.04$, and 0.002 ). Using a cutoff of 5 or more involved LN basins, responders could be separated from nonresponders with a sensitivity, specificity, and positive and negative predictive value of $88 \%, 81 \%, 70 \%$, and $93 \%$, respectively. Using a cutoff of 8.15 for the early $\mathrm{SUV}_{\max }$ of LN basins and of 10 for the late $\mathrm{SUV}_{\max }$ of $\mathrm{LN}$ basins, a comparable sensitivity of $88 \%$ came at the cost of a lower specificity: $73 \%$ and $67 \%$, respectively. ${ }^{139}$ This accuracy tends to be slightly lower on FDG-PET imaging performed at 4 months posttreatment instigation. However, at this time interval, a cutoff for $\mathrm{SUV}_{\max }$ values of involved LNs of 4.5 appears to be more performing: LNs responding to TB treatment could be differentiated from nonresponding LNs with a sensitivity and specificity of 95\% and $85 \%$ (area under the curve: 0.96), respectively. ${ }^{145}$ As well as the number of LNs with rim enhancement and low central attenuation was significantly higher in nonresponders than in

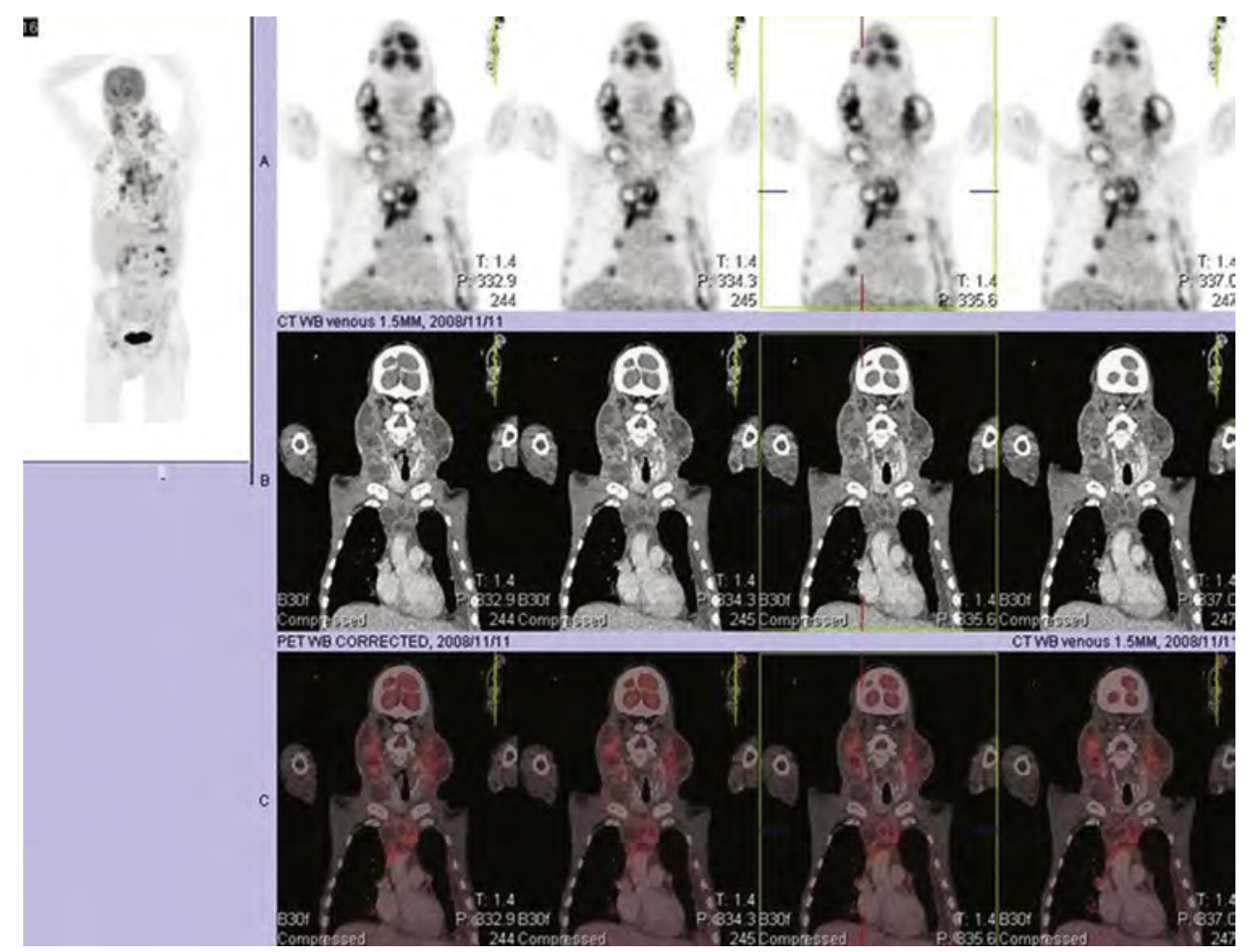

Figure 9 PET/CT findings in a nonresponder with basins of involved lymph nodes displaying central attenuation and peripheral rim enhancement. This support the fact that when the macrophage-activating response is weak, the lesion would enlarge through tissue destruction with central liquefaction of caseous material that contains large numbers of TB bacilli, as shown on CT, corresponding to peripheral rim-enhanced central low-attenuation nodes. 
responders (Fig. 9). All these studies concur that FDG-PET may be a useful tool in the assessment of latent TB to exclude active disease before treatment. PET/CT has the potential for monitoring response to anti-TB treatment. Metabolic response may indicate clinical response and guide duration of antimicrobial therapy.

\section{Conclusions}

The value of PET applied to the metabolic evaluation of HIV and TB is increasingly recognized. With FDG-PET-studies improving our understanding of the pathogenesis of HIV infection and the value in the clinical decision making of HIVinfected patients presenting with AIDS-related opportunistic infections such as TB and malignancies. Knowledge of this varied spectrum of AIDS-defining illnesses and immunovirological status is essential when interpreting FDG-PET imaging. In addition, FDG-PET holds great potential to play a significant role in the clinical decision making of HIV-1 patients presenting with AIDS-related opportunistic infections and malignancies and for to proposing appropriate differential diagnoses, suggest correlation with laboratory and microbiological assays or biopsy, and reliably assess therapeutic response.

\section{References}

1. UNAIDS. Together We Will End AIDS. Geneva, Switzerland: Joint United Nations Programme on HIV/AIDS (UNAIDS); 2012. http:// www.unaids.org/en/resources/campaigns/togetherwewillendaids/

2. WHO. Global Tuberculosis Control 2011. Geneva, Switzerland: World Health Organization; 2011. http://www.who.int/tb/publications/global_report/2011/gtbrl1_full.pdf

3. Kelleher $\mathrm{H}$, Zaunders J: Decimated or missing in action: $\mathrm{CD}^{+} \mathrm{T}$ cells as targets and effectors in the pathogenesis of primary HIV infection. Curr HIV/AIDS Rep 2006;3:5-12

4. Samuel R, Bettiker R, Suh B: AIDS related opportunistic infections, going but not gone. Arch Pharm Res 2002;25:215-228

5. Cadogan M, Dalgleish A: HIV induced AIDS and related cancers: Chronic immune activation and future therapeutic strategies. Adv Cancer Res 2008;101:349-395

6. Aoki Y, Tosato G: Neoplastic conditions in the context of HIV-1 infection. Curr HIV Res 2004;2:343-349

7. Porter C, Laditka J, Cornman C, et al: Prevalence of AIDS dementia complex: Results from a state-wide population-based registry of Alzheimer's disease and related disorders. J S C Med Assoc 2008;104:223-236

8. Naidoo K, Baxter C, Abdool Karim SS: Antiretroviral therapy during tuberculosis treatment. Curr Opin Infect Dis 2013;26:35-42

9. Palella FJ Jr, Baker RK, Moorman AC, et al: Mortality in the highly active antiretroviral therapy era: Changing causes of death and disease in the HIV outpatient study. J Acquir Immune Defic Syndr 2006;43:27-34

10. Sabin CA, Smith CJ, Youle M, et al: Deaths in the era of HAART: Contribution of late presentation, treatment exposure, resistance and abnormal laboratory markers. AIDS 2006;20:67-71

11. Smit C, Geskus R, Walker S, et al: Effective therapy has altered the spectrum of cause-specific mortality following HIV seroconversion. AIDS 2006;20:741-749

12. Crum NF, Riffenburgh RH, Wegner S, et al: Comparisons of causes of death and mortality rates among HIV-infected persons: Analysis of the pre-, early, and late HAART (highly active antiretroviral therapy) eras. J Acquir Immune Defic Syndr 2006;41:194-200

13. Lewden C, May T, Rosenthal E, et al: Changes in causes of death among adults infected by HIV between 2000 and 2005: The "Mortalite 2000 and 2005" surveys (ANRS EN19 and Mortavic). J Acquir Immune Defic Syndr 2008;48:590-598

14. Bonnet F, Lewden C, May T, et al: Opportunistic infections as causes of death in HIV-infected patients in the HAART era in France. Scand J Infect Dis 2005;37:482-487

15. Krentz HB, Kliewer G, Gill MJ: Changing mortality rates and causes of death for HIV-infected individuals living in Southern Alberta, Canada from 1984 to 2003. HIV Med 2005;6:99-106

16. Yang CH, Huang YF, Hsiao CF, et al: Trends of mortality and causes of death among HIV-infected patients in Taiwan, 1984-2005. HIV Med 2008;9:535-543

17. El-Haddad G, Zhuang H, Gupta N, et al: Evolving role of positron emission tomography in the management of patients with inflammatory and other benign disorders. Semin Nucl Med 2004;34: 313-329

18. Kubota R, Kubota K, Yamada S, et al: Microautoradiolgraphic study for the differentiation of intratumoral macrophages, granulation tissues and cancer cells by the dynamics of fluorine-18-fluorodeoxyglucose uptake. J Nucl Med 1994;35:104-112

19. Miceli M, Atoui R, Walker R, et al: Diagnosis of deep septic thrombophlebitis in cancer patients by fluorine-18 fluorodeoxyglucose positron emission tomography scanning: A preliminary report. J Clin Oncol 2004;22:1949-1956

20. Mahfouz T, Miceli MH, Saghafifar F, et al: ${ }^{18}$ F-fluorodeoxyglucose positron emission tomography contributes to the diagnosis and management of infections in patients with multiple myeloma: A study of 165 infectious episodes. J Clin Oncol 2005;23:7857-7863

21. Cloyd M, Chen J, Wang I: How does HIV cause AIDS? The homing theory Mol Med Today 2000;6:108-111

22. Lederman M, Margolis L: The lymph node in HIV pathogenesis. Semin Immunol 2008;20:187-195

23. Bakheet S, Powe J: Benign causes of 18-FDG uptake on whole-body imaging. Semin Nucl Med 2000;28:352-358

24. Brown R, Leung J, Fisher S, et al: Intratumoral distribution of tritiated fluorodeoxyglucose in breast carcinoma: Are inflammatory cells important? J Nucl Med 1995;36:1854-1861

25. Kubota R, Yamada S, Kubota K, et al: Intratumoral distribution of fluorine-18-deoxyglucose in vivo: High accumulation in macrophages and granulation tissues studied by microautoradiography. J Nucl Med 1992;33:1972-1980

26. Bental M, Deutsch C: Metabolic changes in activated Tcells: An NMR study of human peripheral blood lymphocytes. Magn Reson Med 1993;29:317-326

27. Marjanovic S, Skog S, Heiden T, et al: Expression of glycolytic isoenzymes in activated human peripheral lymphocytes: Cell cycle analysis using flow cytometry. Exp Cell Res 1991;193:425-431

28. Kwan A, Seltzer M, Czernin J, et al: Characterization of hilar lymph node by ${ }^{18}$ F-fluro-2-deoxyglucose positron emission tomography in healthy subjects. Anticancer Res 2001;21:701-706

29. Scharko A, Perlman S, Hinds P, et al: Whole body positron emission tomography imaging of simian immunodeficiency virus-infected rhesus macaques. Proc Natl Acad Sci U S A 1996;93:6425-6430

30. Wallace M, Pyzalski R, Horejsh D, et al: Whole body positron emission tomography imaging of activated lymphoid tissues during acute simianhuman immunodeficiency virus 89.6PD infection in rhesus macaques. Virology 2000;274:255-261

31. Scharko A, Perlman S, Pyzalski R, et al: Whole-body positron emission tomography in patients with HIV-1 infection. Lancet 2003;20:959-961

32. Iyengar S, Chin B, Margolick J, et al: Anatomical loci of HIV-associated immune activation and association with viraemia. Lancet 2003;20: 945-950

33. Sathekge M, Maes A, Kgomo M, et al: Fluorodeoxyglucose uptake by lymph nodes of HIV patients is inversely related to CD4 cell count. Nucl Med Commun 2010;31:137-140

34. Brust D, Polis M, Davey R, et al: Fluorodeoxyglucose imaging in healthy subjects with HIV infection: Impact of disease stage and therapy on pattern of nodal activation. AIDS 2006;20:495-503

35. Liu Y: Clinical significance of diffusely increased splenic uptake on FDGPET. Nucl Med Commun 2009;30:763-769 
36. Lucignani G, Orunesu E, Cesari M, et al: FDG-PET imaging in HIVinfected subjects: Relation with therapy and immunovirological variables. Eur J Nucl Med Mol Imaging 2009;36:640-647

37. Durack DT, Street AC: Fever of unknown origin-Reexamined and redefined. Curr Clin Top Infect Dis 1991;11:35-51

38. Knockaert DC, Vanderschueren S, Blockmans D: Fever of unknown origin in adults: 40 years on. J Intern Med 2003;253:263-275

39. Schacker T, Collier AC, Hughes J, et al: Clinical and epidemiologic features of primary HIV infection. Ann Intern Med 1996;125: 257-264

40. De Munter P, Peetermans WE, Derdelinckx I, et al: Fever in HIV-infected patients: Less frequent but still complex. Acta Clin Belg 2012;67: 276-281

41. Koopmans PP, Burger DM: Managing drug reactions to sulfonamides and other drugs in HIV infection: Desensitization rather than rechallenge? Pharm World Sci 1998;20:253-257

42. Bing EG, Burnam MA, Longshore D, et al: Psychiatric disorders and drug use among human immunodeficiency virus-infected adults in the United States. Arch Gen Psychiatry 2001;58:721-728

43. Bleeker-Rovers CP, van der Meer JW, Oyen WJ: Fever of unknown origin. Semin Nucl Med 2009;39:81-87

44. Keidar Z, Gurman-balbir A, Gaitini D, et al: Fever of unknown origin: The role of ${ }^{18}$ F-FDGPET/CT. J Nucl Med 2008;49:1980-1985

45. Glaudemans AWJM, Signore A: FDG-PET/CT in infections: The imaging method of choice? Eur J Nucl Med Mol Imaging 2010;37: 1986-1991

46. Martin C, Castaigne C, Tondeur M, et al: Role and interpretation of FDGPET/CT in HIV patients with fever of unknown origin: A prospective study. J Int AIDS Soc 2012;15(suppl 4):18107

47. Bonnet F, Chêne G: Evolving epidemiology of malignancies in HIV. Curr Opin Oncol 2008;20:534-540

48. Powles T, Robinson D, Stebbing J, et al: Highly active antiretroviral therapy and the incidence of non-AIDS-defining cancers in people with HIV infection. J Clin Oncol 2008;27:884-890

49. Grulich AE, van Leeuwen MT, Falster MO, et al: Incidence of cancers in people with HIV/AIDS compared with immunosuppressed transplant recipients: A meta-analysis. Lancet 2007;370:59-67

50. Bedimo R: Non-AIDS-defining malignancies among HIV-infected patients in the highly active antiretroviral therapy era. Curr HIV/AIDS Rep 2008;5:140-149

51. Crum-Cianflone N, Hullsiek K, Marconi V, et al: Trends in the incidence of cancers among HIV-infected persons and the impact of antiretroviral therapy: A 20-year cohort study. AIDS 2009;2(23):41-50

52. Mayor A, Gomez M, Rios-Olivares E, et al: AIDS-defining neoplasm prevalence in a cohort of HIV-infected patients, before and after highly active antiretroviral therapy. Ethn Dis 2008;18(S2):189-194

53. Chitale A: Cancer and AIDS: Indian. J Pathol Microbiol 2005;48: 151-160

54. Moodley M, Mould S: Invasive cervical cancer and human immunodeficiency virus (HIV) infection in KwaZulu-Natal, South Africa. J Obstet Gynecol 2005;25:706-710

55. Kidd EA, Grigsby PW: Intratumoral metabolic heterogeneity of cervical cancer. Clin Cancer Res 2008;14:5236-5241

56. Einstein $\mathrm{MH}$, Phaeton R: Issues in cervical cancer incidence and treatment in HIV. Curr Opin Oncol 2010;22:449-455

57. Grigsby PW: Role of PET in gynecologic malignancy. Curr Opin Oncol 2009;21:420-424

58. Kidd EA, El Naqa I, Siegel BA, et al: FDG-PET-based prognostic nomograms for locally advanced cervical cancer. Gynecol Oncol 2012;127:136-140

59. Schwartz EJ, Dorfman RF, Kohler S: Human herpesvirus-8 latent nuclear antigen-1 expression in endemic Kaposi sarcoma: An immunohistochemical study of 16 cases. Am J Surg Pathol 2003;27:1546-1550

60. Sullivan R, Dezube BJ, Koon HB: Signal transduction targets in Kaposi's sarcoma. Curr Opin Oncol 2006;18:456-462

61. Bower M, Palmieri C, Dhillon T: AIDS-related malignancies: Changing epidemiology and the impact of highly active antiretroviral therapy. Curr Opin Infect Dis 2006;19:14-19
62. van de Luijtgaarden A, van der Ven A, Leenders W, et al: Imaging of HIVassociated Kaposi sarcoma. F-18-FDG-PET/CT and In-111bevacizumabscintigraphy. J AIDS 2010;54:444-446

63. Morooka M, Ito K, Kubota K, et al: Whole-body ${ }^{18} \mathrm{~F}$-fluorodeoxyglucose positron emission tomography/computed tomography images before and after chemotherapy for Kaposi sarcoma and highly active antiretrovirus therapy. Jpn J Radiol 2010;28:759-762

64. Krown SE, Testa MA, Huang J: AIDS-related Kaposi's sarcoma: Prospective validation of the AIDS Clinical Trials Group staging classification. AIDS Clinical Trials Group Oncology Committee. J Clin Oncol 1997;15:3085-3092

65. Dupin N, Diss TL, Kellam P, et al: HHV-8 is associated with a plasmablastic variant of Castleman disease that is linked to HHV-8positive plasmablastic lymphoma. Blood 2000;95:1406-1412

66. Barker R, Kazmi F, Stebbing J, et al: FDG-PET/CT imaging in the management of HIV-associated multicentric Castleman's disease. Eur J Nucl Med Mol Imaging 2009;36:648-652

67. Engels EA, Pfeiffer RM, Goedert JJ, et al: Trends in cancer risk among people with AIDS in the United States 1980-2002. AIDS 2006;20:1645

68. Diamond C, Taylor TH, Aboumrad T, et al: Changes in acquired immunodeficiency syndrome-related non-Hodgkin lymphoma in the era of highly active antiretroviral therapy. Cancer 2005;106: 128-135

69. Engels EA, Pfeiffer RM, Landgren O, et al: Immunologic and virologic predictors of AIDS-related non-Hodgkin lymphoma in the highly active antiretroviral therapy era. J Acquir Immune Defic Syndr 2010;54: 78-84

70. Guiguet M, Boué F, Cadranel J, et al: Effect of immunodeficiency, HIV viral load, and antiretroviral therapy on the risk of individual malignancies (FHDH-ANRS CO4): A prospective cohort study. Lancet Oncol 2009;10:1152-1159

71. Zoufaly A, Stellbrink HJ, Heiden MA, et al: Cumulative HIV viremia during highly active antiretroviral therapy is a strong predictor of AIDSrelated lymphoma. J Infect Dis 2009;200:79-87

72. Guech-Ongey M, Simard EP, Anderson WF, et al: AIDS-related Burkitt lymphoma in the United States: What do age and CD4 lymphocyte patterns tell us about etiology and/or biology? Blood 2010;116:5600-5604

73. Gabarre J, Raphael M, Lepage E, et al: Human immunodeficiency virusrelated lymphoma: Relation between clinical features and histologic subtypes. Am J Med 2001;111:704-711

74. Forsyth PA, DeAngelis LM: Biology and management of AIDS-associated primary CNS lymphomas. Hematol Oncol Clin North Am 1996;10: 1125-1134

75. Franceschi S, Lise M, Clifford GM, et al: Changing patterns of cancer incidence in the early- and late-HAART periods: The Swiss HIV Cohort Study. Br J Cancer 2010;103:416-422

76. Liu Y: Demonstrations of AIDS-associated malignancies and infections at FDG PET-CT. Ann Nucl Med 2011;25:536-546

77. Goshen E, Davidson T, Avigdor A, et al: PET/CT in the evaluation of lymphoma in patients with HIV-1 with suppressed viral loads. Clin Nucl Med 2008;33:610-614

78. Sathekge M, Maes A, Kgomo M, et al: FDG uptake in lymph-nodes of $\mathrm{HIV}+$ and tuberculosis patients: Implications for cancer staging. Q J Nucl Med Mol Imaging 2010;54:698-703

79. Just $P$, Fieschi $C$, Baillet $G$, et al: ${ }^{18}$ F-fluorodeoxyglucose positron emission tomography/computed tomography in AIDS-related Burkitt lymphoma. Aids Patient Care STDS 2008;22:695-700

80. Martis H, Mounier N: Hodgkin lymphoma in patients with HIV infection: A review. Curr Hematol Malig Rep 2012;7:228-234

81. Hoffman JM, Waskin HA, Schifter T, et al: FDG-PET in differentiating lymphoma from nonmalignant central nervous system lesions in patients with AIDS. J Nucl Med 1993;34:567-575

82. Villringer K, Jager H, Dichgans M, et al: Differential diagnosis of CNS lesions in AIDS patients by FDG-PET. J Comput Assist Tomogr 1995; 19:532-536

83. Heald A, Hoffman JM, Bartlett J, et al: Differentiation of central nervous system lesions in AIDS patients using positron emission tomography (PET). Int J STD AIDS 1996;7:337-346 
84. O'Doherty M, Barrington S, Campbell M, et al: PET scanning and the human immunodeficiency virus-positive patient. J Nucl Med 1997;38: 1575-1583

85. Woods SP, Moore DJ, Weber E, et al: Cognitive neuropsychology of HIV-associated neurocognitive disorders. Neuropsychol Rev 2009;19: 152-1568

86. Hammoud DA, Hoffmann JM, Pomper MG: Molecular neuroimaging: From conventional to emerging techniquesl. Radiology 2007;24:21-42

87. Boska M, Mosley R, Nawab M, et al: Advances in neuroimaging for HIV1 associated neurological dysfunction: Clues to the diagnosis, pathogenesis and therapeutic monitoring. Curr HIV Res 2004;2:61-78

88. Depas G, Chiron C, Tardieu M, et al: Functional brain imaging in HIV-1infected children born to seropositive mothers. J Nucl Med 1995;36: 2169-2174

89. Hinkin C, Van Gorp W, Mandelkern M, et al: Cerebral metabolic change in patients with AIDS: Report of a six-month follow-up using positronemission tomography. J Neuropsychiatry Clin Neurosci 1995;7: 180-187

90. Newton T, Leuchter A, Walter D, et al: EEG coherence in men with AIDS: Association with subcortical metabolic activity. J Neuropsychiatry Clin Neurosci 1993;5:316-321

91. Rottenberg D, Sidtis J, Strother S, et al: Abnormal cerebral glucose metabolism in HIV-1 seropositive subjects with and without dementia. J Nucl Med 1996;37:1133-1141

92. Villemagne V, Phillips R, Liu X, et al: Peptide T and glucose metabolism in AIDS dementia complex. J Nucl Med 1996;37:1177-1180

93. von Giesen H, Antke C, Hefter H, et al: Potential time course of human immunodeficiency virus type 1-associated minor motor deficits: Electrophysiologic and positron emission tomography findings. Arch Neurol 2000;57:1601-1607

94. Rottenberg D, Moeller J, Strother S, et al: The metabolic pathology of the AIDS dementia complex. Ann Neurol 1987;22:700-706

95. Van Gorp W, Mandelkern M, Gee M, et al: Cerebral metabolic dysfunction in AIDS: Findings in a sample with and without dementia. J Neuropsychiatry Clin Neurosci 1992;4:280-287

96. Kingsley LA, Cuervo-Rojasc J, Muñoz A, et al: Subclinical coronary atherosclerosis, HIV infection and antiretroviral therapy: Multicenter AIDS Cohort Study. AIDS 2008;22:1589-1599

97. Baker JV, Henry WK, Neaton JD: The consequences of HIV infection and antiretroviral therapy use for cardiovascular disease risk: Shifting paradigms. Curr Opin HIV AIDS 2009;4:176-182

98. Stein JH: Cardiovascular risks of antiretroviral therapy. N Engl J Med 2007;356:1773-1775

99. Hoh CK: Clinical use of FDG PET. Nucl Med Biol 2007;34:737-742

100. Hongming Z, Abass A: 18-Fluorodeoxyglucose positron emission tomographic imaging in the detection and monitoring of infection and inflammation. Semin Nucl Med 2002;32:47-59

101. Fox JJ, Strauss HW: One step closer to imaging vulnerable plaque in the coronary arteries. J Nucl Med 2009;50:497-500

102. Yarasheski KE, Laciny E, Overton ET, et al: ${ }^{18}$ FDG PET-CT imaging detects arterial inflammation and early atherosclerosis in HIV-infected adults with cardiovascular disease risk factors. J Inflamm (Lond) 2012;9:26

103. Subramanian S, Tawakol A, Burdo TH, et al: Arterial inflammation in patients with HIV. J Am Med Assoc 2012;308:379-386

104. Carr A: Diagnosis, prediction and natural course of HIV-1 protease inhibitor-associated lipodystrophy, hyperlipidemaemia, and diabetes mellitus: A cohort study. Lancet 1999;353:2093-2099

105. Behrens G, Stoll M, Schmidt R: Lipdosystrophy syndrome in HIV infection: What is it, what causes it and how can it be managed? Drug Saf 2000; 23: 57-76

106. Behrens G, Boerner A, Weber K, et al: Impaired glucose phosphorylation and transport in skeletal muscle cause insulin resistance in HIV-1infected patients with lipodystrophy. J Clin Invest 2002;110: $1319-1327$

107. Bleeker-Rovers C, van der Ven A, Zomer B, et al: F-18-Fluorodexoyglucose positron emission tomography for visualization of lipodystrophy in HIV-infected patients. AIDS 2004;18:2430-2432
108. Sathekge M, Maes A, Kgomo M, et al: Evaluation of glucose uptake by skeletal muscle tissue and subcutaneous fat in HIV-infected patients with and without lipodystrophy using FDG-PET. Nucl Med Commun 2010;31:311-314

109. Warwick JM, Sathekge MM: PET/CT scanning with a high HIV/AIDS prevalence. Transfus Apher Sci 2011;44:167-172

110. Tsong Fang H, Colantonio A, Uittenbogaart C: The role of the thymus in HIV infection: A 10 year perspective. AIDS 2008;22: 171-174

111. Hardy G, Worrell S, Hayes P, et al: Evidence of thymic reconstitution after highly active antiretroviral therapy in HIV-1 infection. HIV Med 2004;5:67-73

112. Tanaskovic S, Fernandez S, French MA, et al: Thymic tissue is not evident on high-resolution computed tomography and $\left[{ }^{18} \mathrm{~F}\right]$ fluorodeoxy-glucose positron emission tomography scans of aviraemic HIV patients with poor recovery of $\mathrm{CD}^{+}$T cells. AIDS 2011;25: $1235-1237$

113. Zou W, Foussat A, Houhou S: Acute upregulation of CCR-5 expression by $\mathrm{CD}^{+} \mathrm{T}$ lymphocytes in HIV-infected patients treated with interleukin-2. AIDS 1999;13:455-463

114. World Health Organization. Global Tuberculosis Control Report. Surveillance Planning Financing. Geneva: WHO; 2009

115. Sathekge M, Buscombe JR: Can positron emission tomography work in the African tuberculosis epidemic? Nucl Med Commun 2011;32: 241-244

116. UNAIDS. Report on the Global AIDS Epidemic. Geneva: UNAIDS; 2008

117. Mendelson M: Diagnosing tuberculosis in HIV-infected patients: Challenges and future prospects. Br Med Bull 2007;81-82:149-165

118. Kosterink JGW: Positron emission tomography in the diagnosis and treatment management of tuberculosis. Curr Pharm Des 2011;17: 2875-2880

119. Zhuang H, Alavi A: 18-Fluorodeoxyglucose positron emission tomographic imaging in the detection and monitoring of infection and inflammation. Semin Nucl Med 2002;32:47-59

120. Matthies A, Hickeson M, Cuchiara A, et al: Dual point ${ }^{18}$ FFDG PET for the evaluation of pulmonary nodules. J Nucl Med 2002;43: 871-875

121. Goo J, Im H, Do K, et al: Pulmonary tuberculoma evaluated by means of FDG PET: Findings in 10 cases. Radiology 2000;216:117-121

122. Kim I, Lee J, Kim S, et al: Double-phase ${ }^{18}$ F-FDG PET-CT for determination of pulmonary tuberculoma activity. Eur J Nucl Med Mol Imaging 2008;35:808-814

123. Cameron R: Inflammation and repair. In: Robbins SL, (ed): Pathology. ed 3. Philadelphia, PA: WB Saunders; 1967. pp. 31-37

124. Adams D: The granulomatous inflammatory response: A review. Am J Pathol 1976;84:161-191

125. Dannenberg A, Tamashefski J: Pathogenesis of pulmonary tuberculosis. In: Fishman A, Elias J, Fishman J, et al.,(eds): Fishman's Pulmonary Disease and Disorders. ed 3. New York, NY: McGraw-Hill; 1998. pp. 2447-2471

126. Cazin M, Paluszezal D, Bianchi A, et al: Effects of anaerobosis upon morphology and energy metabolism of alveloar macrophages cultured in gas phase. Eur Respir J 1990;3:1015-1022

127. Hara $\mathrm{T}$, Kosaka $\mathrm{N}$, Suzuki $\mathrm{T}$, et al: Uptake rates of ${ }^{18} \mathrm{~F}$-fluorodeoxyglucose and ${ }^{11} \mathrm{C}$-choline in lung cancer and pulmonary tuberculosis. Chest 2003;124:893-901

128. Lan XL, Zhang YX, Wu ZJ, et al: The value of dual time point ${ }^{18} \mathrm{~F}-\mathrm{FDG}$ PET imaging for the differentiation between malignant and benign lesions. Clin Radiol 2008;63:756-764

129. Chen CJ, Lee BF, Yao WJ, et al: Dual-phase ${ }^{18}$ F-FDG PET in the diagnosis of pulmonary nodules with an initial standard uptake value less than 2.5. AJR Am J Roentgenol 2008;191:475-479

130. Sathekge MM, Maes A, Pottel H, et al: Dual time-point FDG PET-CT for differentiating benign from malignant solitary pulmonary nodules in a TB endemic area. S Afr Med J 2010;100:598-601

131. WHO (World Health Organization): Global Tuberculosis Control: WHO Report. Geneva, 2011. http://www.who.int/tb/publications/global _report/en/ 
132. WHO (World Health Organization): Global Tuberculosis Control: surveillance, Planning, Financing. WHO Report, Geneva, 2007. http://www.who.int/tb/publications/global_report/2007/ pdf/full. pdf

133. Peto HM, Pratt RH, Harrington TA, et al: Epidemiology of extrapulmonary tuberculosis in the United States, 1993-2006. Clin Infect Dis 2009; 49:1350-1357

134. Sharma S, Mohan A: Extrapulmonary tuberculosis. Indian J Med Res 2004;120:316-353

135. Arciniegas W, Orjuela D: Extrapulmonary tuberculosis: A review of 102 cases in Pereira, Colombia. Biomedica 2006;26:71-80

136. Golden M, Vikram H: Extrapulmonary tuberculosis: An overview. Am Fam Physician 2005;72:1761-1768

137. Rivas-Garcia A, Sarria-Estrada S, Torrents-Odin C, et al: Imaging findings of Pott's disease. Eur Spine J 2012. [Epub ahead of print]

138. Sathekge M, Maes A, Kgomo M, et al: Impact of FDG PET on the management of TBC treatment. A pilot study. Nuklearmedizin 2010;49: $35-40$

139. Sathekge M, Maes A, Kgomo M, et al: Use of ${ }^{18}$ F-FDG PET to predict response to first-line tuberculostatics in HIV-associated tuberculosis. J Nucl Med 2011;52:1-6
140. Davis SL, Nuermberger EL, Um PK, et al: Noninvasive pulmonary $\left[{ }^{18} \mathrm{~F}\right]$ 2-fluoro-deoxy-D-glucose positron emission tomography correlates with bactericidal activity of tuberculosis drug treatment. Antimicrob Agents Chemother 2009;53:4879-4884

141. Demura Y, Tsuchida T, Uesaka D: Usefulness of ${ }^{18} \mathrm{~F}$-fluorodeoxyglucose positron emission tomography for diagnosing disease activity and monitoring therapeutic response in patients with pulmonary mycobacteriosis. Eur J Nucl Med Mol Imaging 2009;36:632-639

142. Park I, Ryu J, Sun Shim T: Evaluation of therapeutic response of tuberculoma using F-18 FDG positron emission tomography. Clin Nucl Med 2008;33:1-3

143. Tian G, Xiao Y, Chen B, et al: FDG PET/CT for therapeutic response monitoring in multi-site non-respiratory tuberculosis. Acta Radiol 2010;51:1002-1006

144. Hofmeyr A, Lau E, Slavin M: Mycobacterium tuberculosis infection in patients with cancer, the role of 18-fluorodeoxyglucose positron emission tomography for diagnosis and monitoring treatment response. Tuberculosis 2007;87:459-463

145. Sathekge M, Maes A, D'Asseler Y, et al: Tuberculous lymphadenitis: FDG PET and CT findings in responsive and nonresponsive disease. Eur J Nucl Med Mol Imaging 2012;39:1184-1190 